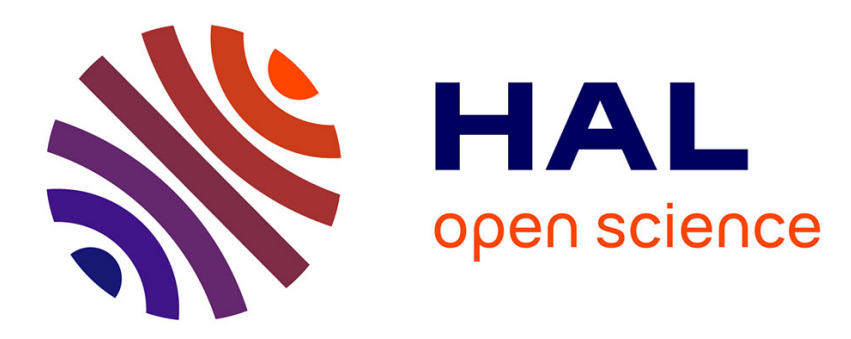

\title{
B-Spline interpolation of Kirchhoff-Love space rods
}

Leopoldo Greco, Massimo Cuomo

\section{To cite this version:}

Leopoldo Greco, Massimo Cuomo. B-Spline interpolation of Kirchhoff-Love space rods. Computer Methods in Applied Mechanics and Engineering, 2013, 256, pp.251-269. hal-00874611

\section{HAL Id: hal-00874611 https://hal.science/hal-00874611}

Submitted on 18 Oct 2013

HAL is a multi-disciplinary open access archive for the deposit and dissemination of scientific research documents, whether they are published or not. The documents may come from teaching and research institutions in France or abroad, or from public or private research centers.
L'archive ouverte pluridisciplinaire HAL, est destinée au dépôt et à la diffusion de documents scientifiques de niveau recherche, publiés ou non, émanant des établissements d'enseignement et de recherche français ou étrangers, des laboratoires publics ou privés. 


\title{
B-Spline interpolation of Kirchhoff-Love space rods
}

\author{
L. Greco, M. Cuomo
}

Department of Civil and Environmental Engineering, Universitá degil Studi di Catania, viaie A. Doria 6, itaty

\begin{abstract}
A B S T R A C T
The paper deals with the isogeometric analysis via B splines of space rods under Kirchhoff Love hypoth eses. The approach was used by Gontier and Vollmer [12] for developing a plane curve element within the framework of the Timoshenko rod model, but they adopted only one patch to represent entirely the geometry of the rod; furthermore the authors developed their theory only for plane elements. In this work we develop an isogeometric approach for the numerical analysis of the 3D Kirchhoff Love rod the ory. We use B splines and Bezier interpolations and we show that they are able to attain very good accu racy for rod structures, particularly for developing a 3D exact curve element with geometric torsion. The paper presents an original parametrization of the geometric torsion that proves to be very effective. The use of B splines allows to avoid discontinutities on the geometrical quantities, and particularly on the nor mal fields, so that even relatively low order interpolation functions are able to yield accurate results.
\end{abstract}

\section{Introduction}

Isogeometric analysis is a new paradigm in computational mechanics, mainly introduced by Hughes et al. [14], that employs the geometrical representation tools developed in the computer aided design, and in the computer graphics technology. In isogeo metric analysis the geometrical objects are interpolated with B splines, which guarantee $C^{p}$ continuity, $p$ being the degree of the spline, as opposite to the usual $C^{0}$ continuity obtained with the standard FEM discrete representations. The same interpolation is used for the degrees of freedom that define the deformed geom etry, so that an isoparametric description is obtained. Although B splines are not shape functions in the usual sense, they verify the partition of unity.

An extensive review of B splines technology can be found in Piegl and Tiller [21]. In this work we employ open B splines defined on an uniform knots vector.

Recently isogeometric analysis has been applied to many prob lems of solid and fluid mechanics [17]. Its ability to correctly incor porate in the analysis the initial geometric curvatures without discontinuities makes the method very appealing for rods and shells. Isogeometric analysis of shell models has been developed by [6] for polar and by Benson et al. [5] and Kiendl et al. [18] for non polar shells. In [6] the authors pay particular attention to the interpolation of the normal vector, introducing a method based on a newly proposed lifting operator. In [19] a procedure for join ing different patches under Kirchhoff Love hypotheses is proposed.

In the field of 1D structural theories it is interesting to point out that already in 1995 Gontier and Vollmer [12] applied a Bezier's interpolation to a plane polar rod model on the basis of the theory of Simo [22]. Recently Echter and Bischoff [11] have studied a low order Bezier's interpolation for polar beams, in order to investigate the numerical shear locking. They also developed an interesting generalization of the DSG approach for avoiding locking to the case of isogeometric analysis, extending the idea of Bletzinger et al. [8] of the Discrete Strain Gap. Only the case of straight beams was cov ered by the authors.

A general model for space rods has been given by Simo and Vu Quoc [24] who also provided its numerical implementation. However they adopted a Timoshenko model. The bibliography on rods is huge, and we only cite the significant numerical contribu tions given by Crisfield [10], and, for Kirchhoff models by Goriely et al. [13], Langer and Singer [20], Antman [1]. Finite Element implementations of the Kirchhoff Love model for finite deforma tion space rods are not common in literature, since continuity con ditions for the geometry are more difficult to enforce. However, Kirchhoff Love model has some advantages, mostly related to the absence of shear locking phenomena especially for slender and thin walled elements, and to the greater compactness of the for mulation. Furthermore, the exact evaluation of the shear correction factor is still a matter of discussion.

In the context of standard polynomial interpolations of Finite Elements many elements have been proposed for effectively treat ing this kind of structures, generally based on mixed or enhanced formulations $[24,10,2]$. Special elements for particular geometries 
have been proposed by Ishaquddin et al. [16]. In this work we adopt a pure displacement $B$ Spline isoparametric formulation of a space rod model. It is shown that the model can be efficiently implemented in a numerical code, and that the use of a smooth geometry improves the convergence rate of the solution. Since we do not use NURBS, the reference geometry may be affected by errors, so it is proposed a refinement strategy that improves both the approximation of the reference geometry and of the con figuration variables, similarly to what happens in FEM. It will be shown that, at least for the case examined, there is no appreciable loss of precision with respect to the NURBS approximation.

We consider a parametrization of the cross section independent from the Frenet's triad, similar to the natural frame and curvilinear angle representation given in [20]. The normal vectors attached to the centroid curve are mapped by a correction angle, $\phi$, that coin cides with the torsional twist. The geometry of the axis is defined via a uniform collocation of the control points. The tangent and normal vectors are defined on the interpolation of the geometry. In this work we consider only a single patch to represent the entire geometry of the rod. For the quadrature we adopt the standard Gauss Lobatto rules, considering $p+1$ quadrature's points for each non null section in the knots vector, (where $p$ is the polynomial degree). Alternative strategy of quadrature rules for NURBS inter polations can be found in [15,3].

In the paper the finite deformation kinematics is derived, and the full non linear equilibrium equations are stated. However, since the main goal of the work is to analyze the performance of the B spline approximation, in the applications we have examined only the case of infinitesimal deformation. It is consistently obtained linearizing the finite deformation kinematics for the space rod. The extension to geometric non linear problems will be considered in a forthcoming paper.

\section{Configuration of the space rod}

The rod is treated as a ribbon, defined as a pair $\mathcal{A}, \hat{\boldsymbol{n}}$ where $\mathcal{A}] 0, L_{0}$ [ is an open set of $\mathbb{R}$, that is the parametric domain of the curve $\boldsymbol{p}(S): \mathcal{A} \rightarrow \mathbb{R}^{3}$ and $\hat{\boldsymbol{n}}(S): \mathcal{A} \rightarrow \mathbb{R}^{3}$ is a unit vector field everywhere orthogonal to that curve. An index ' 0 ' denotes the ini tial undeformed configuration. The normal to the original configu ration $\hat{\boldsymbol{n}}_{0}$ is chosen arbitrarily, i.e. it does not coincide with the Frenet normal. The tangent vector to the reference configuration of the curve is the unit vector field $\hat{\boldsymbol{t}}_{0}: \mathcal{A} \rightarrow \mathbb{R}^{3}$ with $\hat{\boldsymbol{t}}_{0} \quad \frac{d p_{0}}{d S}$ (a hat indicates a unit vector). Then there exists a rotation operator $\Lambda\left(\hat{\boldsymbol{t}}_{0}(0), \hat{\boldsymbol{t}}_{0}(S)\right)$ that transforms $\hat{\boldsymbol{t}}_{0}(0)$ in $\hat{\boldsymbol{t}}_{0}(S)$ without rotation around $\hat{\boldsymbol{t}}_{0}(0)$ (its explicit form is given in Eq. (8)). The vector field

$\hat{\boldsymbol{n}}_{0}^{b}(S) \quad \boldsymbol{\Lambda}\left(\hat{\boldsymbol{t}}_{0}(0), \hat{\boldsymbol{t}}_{0}(S)\right) \hat{\boldsymbol{n}}_{0}(0)$

defines the manifold $\mathcal{S}_{0}^{b} \quad\left\{\left(\boldsymbol{p}_{\mathbf{0}}(S), \hat{\boldsymbol{n}}_{0}^{b}(S)\right)\right\}$ that is a geodetic ribbon in the sense of a torsion free ribbon.

The geodetic ribbon is only a particular kind of admissible con figuration. The more general configuration of the space rod is ob tained allowing a torsion around the tangent axis, so that the unit normal to the rod axis is given by the isometric operator

$$
\begin{aligned}
\hat{\boldsymbol{n}}_{0}(S) & \boldsymbol{R}\left(\hat{\boldsymbol{t}}_{0}(S), \phi_{0}(S)\right) \hat{\boldsymbol{n}}_{0}^{b}(S) \\
& \boldsymbol{R}\left(\hat{\boldsymbol{t}}_{0}(S), \phi_{0}(S)\right) \boldsymbol{\Lambda}\left(\hat{\boldsymbol{t}}_{0}(0), \hat{\boldsymbol{t}}_{0}(S)\right) \hat{\boldsymbol{n}}_{0}(0) .
\end{aligned}
$$

where the unit operator $\boldsymbol{R}\left(\hat{\boldsymbol{t}}_{0}(S), \phi_{0}(S)\right)$, represents a rotation $\phi_{0}$ around the unit tangent $\hat{\boldsymbol{t}}_{0}(S)$.

The space rod is thus defined by two fields,

$\mathcal{S}_{0} \quad\left\{\left(\boldsymbol{p}_{\mathbf{0}}(S), \phi_{0}(S)\right): \mathcal{A} \rightarrow \mathbb{R}^{3} \times \mathbb{S}^{1}\right\}$,

The unit local triad is completed by the unit vector

$$
\hat{\boldsymbol{v}}_{0}(S) \quad \hat{\boldsymbol{t}}_{0}(S) \times \hat{\boldsymbol{n}}_{0}(S)
$$

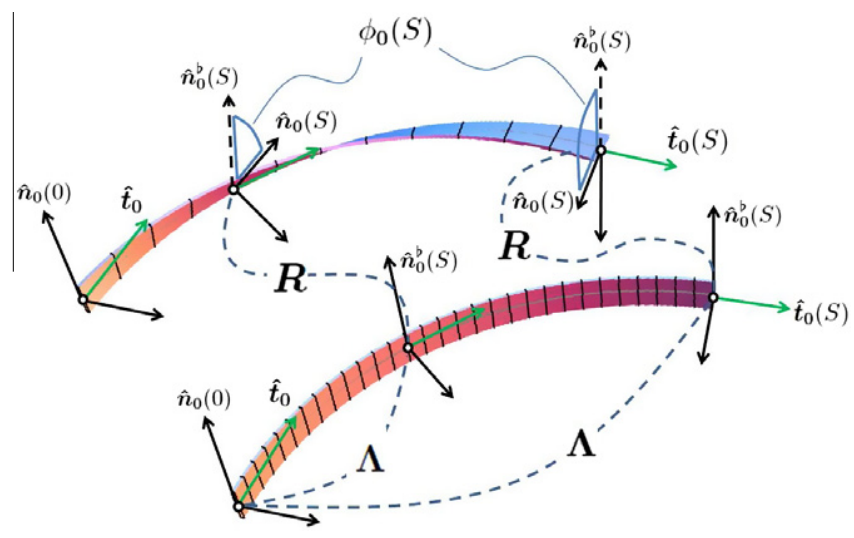

Fig. 1. Intrinsic reference axes on the initial geometry of the rod.

The geometrical transformation (2) defines the more general Lagrangian configuration of a rod, i.e. a rod affected by geometrical torsion but with directors always orthogonal to the axis (see Fig. 1).

\section{Kinematics of the Kirchhoff Love rod}

The space rod model has four degrees of freedom while there are six components of the displacement of the cross section. In Sec tion 3.1 the relationships between internal and external degrees of freedom will be derived.

\subsection{Internal mapping}

Let $\boldsymbol{p}_{0}(S):\left[0, L_{0}\right] \rightarrow \mathbb{R}^{3}$ be the arc length parametrization of the base curve of the reference configuration, and let $\boldsymbol{u}(S):\left[0, L_{0}\right] \rightarrow \mathbb{R}^{3}$ be the Lagrangian arc length parametrization of the displacement field of the centroid curve of the rod. The current centroid curve is indicated by $\boldsymbol{p}(S):\left[0, L_{0}\right] \rightarrow \mathbb{R}^{3}$ and is defined as

$\boldsymbol{p}(S) \quad \boldsymbol{p}_{0}(S)+\boldsymbol{u}(S)$.

The Lagrangian arc length parametrization of the current tan gent vector field is indicated by $\boldsymbol{t}(S):\left[0, L_{0}\right] \rightarrow \mathbb{R}^{3}$ and is defined by $\boldsymbol{t}(S) \frac{d \boldsymbol{p}}{d S}$, so that the current unit tangent vector field $\hat{\boldsymbol{t}}(S):\left[0, L_{0}\right] \rightarrow \mathbb{R}^{3}$ is

$\hat{\boldsymbol{t}}(S) \frac{\boldsymbol{t}(S)}{\|\boldsymbol{t}(S)\|} \quad \frac{1}{\|\boldsymbol{t}(S)\|} \frac{d \boldsymbol{p}}{d S}$.

The intrinsic triad is mapped by means of two isometric operators $\boldsymbol{\Lambda}\left(\hat{\boldsymbol{t}}_{0}(S), \hat{\boldsymbol{t}}(S)\right)$, a rotation without drilling rotation around the vector $\hat{\boldsymbol{t}}_{0}(S)$, and a second unitary operator $\boldsymbol{R}(\hat{\boldsymbol{t}}(S), \phi(S))$ that gives the cor rection drilling rotation $\phi(S):\left[0, L_{0}\right] \rightarrow \mathbb{R}$ around the vector $\hat{\boldsymbol{t}}(S)$.

The proposed parametrization of the triad's rotation differs from the one originally proposed by Simo and Vu Quoc [24], and extensively used since, based on Euler angles around the material or spatial axes. In a Kirchhoff Love model the latter parametriza tion is not convenient, since only one rotation is independent. Lan ger and Singer [20] introduced for Kirchhoff Love rods a natural frame, characterized by having uniformly zero twist (Bishop frame), defined on the rod axis starting from a frame arbitrarily introduced in one point of the curve (usually the point $S=0$ ). The idea was systematically exploited by Bergou et al. [7] for uncoupling the bending and the torsion energy of inextensible Kir chhoff Love rods. This parametrization requires, in addition to the map of the twist angle, the rotation of the frame at $S=0$, that may be not convenient when dealing with rods linked at their end points. The parametrization of the frame rotation proposed here is different, in that no initial value of the spatial vectors is required, and the rotation is always referred to the original configuration. 
The frame we obtain is not "natural" in Langer and Singer sense, as it will be shown soon. However, it appears that the proposed description of the kinematics is simpler and more general, and is easily extended to the case of extensible rods.

Specifically, the two rotation operators are obtained particular izing Euler Rodriguez formula

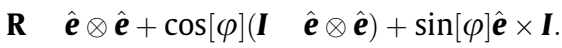

The unitary axial vector of the first rotation is $\hat{\boldsymbol{e}} \frac{\hat{\boldsymbol{t}}_{0} \times \hat{\boldsymbol{t}}}{\left\|\boldsymbol{t}_{0} \times \boldsymbol{t}\right\|}$ while $\cos [\varphi] \quad \hat{\boldsymbol{t}}_{0} \quad \hat{\boldsymbol{t}}$ and $\sin [\varphi] \quad\left\|\hat{\boldsymbol{t}}_{0} \times \hat{\boldsymbol{t}}\right\|$, therefore the formula (7) gives the representation

$\boldsymbol{\Lambda}\left(\hat{\boldsymbol{t}}_{0}, \hat{\boldsymbol{t}}\right) \quad\left(\hat{\boldsymbol{t}}_{0} \cdot \hat{\boldsymbol{t}}\right) \boldsymbol{I}+\left[\hat{\boldsymbol{t}}_{0} \times \hat{\boldsymbol{t}}\right] \times \boldsymbol{I}+\frac{1}{1+\hat{\boldsymbol{t}}_{0} \cdot \hat{\boldsymbol{t}}}\left(\hat{\boldsymbol{t}}_{0} \times \hat{\boldsymbol{t}}\right) \otimes\left(\hat{\boldsymbol{t}}_{0} \times \hat{\boldsymbol{t}}\right)$.

The axial vector of the second rotation operator is $\hat{\boldsymbol{e}} \hat{\boldsymbol{t}}$. Setting $\varphi=\phi$ and using the following property of the double cross product:

$\hat{\boldsymbol{t}} \times[\hat{\boldsymbol{t}} \times \boldsymbol{I}] \quad \boldsymbol{I}+\hat{\boldsymbol{t}} \otimes \hat{\boldsymbol{t}}$

or the equivalent

$\hat{\boldsymbol{t}} \otimes \hat{\boldsymbol{t}} \quad \boldsymbol{I}+\hat{\boldsymbol{t}} \times[\hat{\boldsymbol{t}} \times \boldsymbol{I}]$.

the Rodriguez operator (7) assumes the representation

$\boldsymbol{R}(\hat{\boldsymbol{t}}, \phi) \quad \boldsymbol{I}+\sin [\phi] \hat{\boldsymbol{t}} \times \boldsymbol{I}+(1 \quad \cos [\phi]) \hat{\boldsymbol{t}} \times[\hat{\boldsymbol{t}} \times \boldsymbol{I}]$.

Introducing the definition

$\boldsymbol{Q}\left(\hat{\boldsymbol{t}}_{0}(S), \hat{\boldsymbol{t}}(S), \phi(S)\right) \quad \boldsymbol{R}(\hat{\boldsymbol{t}}(S), \phi(S)) \boldsymbol{\Lambda}\left(\hat{\boldsymbol{t}}_{0}(S), \hat{\boldsymbol{t}}(S)\right)$.

the Lagrangian parametrization of the current directors $\hat{\boldsymbol{n}}(S)$ and $\hat{\boldsymbol{v}}(S)$ are

$$
\begin{array}{lllll}
\hat{\boldsymbol{n}}(S) & \boldsymbol{Q}\left(\hat{\boldsymbol{t}}_{0}, \hat{\boldsymbol{t}}, \phi\right) \hat{\boldsymbol{n}}_{0}(S) & \boldsymbol{R}(\hat{\boldsymbol{t}}, \phi) \hat{\boldsymbol{n}}^{\mathrm{b}}(S), & \hat{\boldsymbol{n}}^{\mathrm{b}}(S) & \boldsymbol{\Lambda}\left(\hat{\boldsymbol{t}}_{0}, \hat{\boldsymbol{t}}\right) \hat{\boldsymbol{n}}_{0}(S), \\
\hat{\boldsymbol{v}}(S) & \boldsymbol{Q}\left(\hat{\boldsymbol{t}}_{0}, \hat{\boldsymbol{t}}, \phi\right) \hat{\boldsymbol{v}}_{0}(S) & \boldsymbol{R}(\hat{\boldsymbol{t}}, \phi) \hat{\boldsymbol{v}}^{b}(S), & \hat{\boldsymbol{v}}^{b}(S) & \boldsymbol{\Lambda}\left(\hat{\boldsymbol{t}}_{0}, \hat{\boldsymbol{t}}\right) \hat{\boldsymbol{v}}_{0}(S) .
\end{array}
$$

We observe that it is also possible to define the $\boldsymbol{Q}$ operator as $\boldsymbol{Q}\left(\hat{\boldsymbol{t}}_{0}, \hat{\boldsymbol{t}}, \phi\right) \quad \boldsymbol{\Lambda}\left(\hat{\boldsymbol{t}}_{0}, \hat{\boldsymbol{t}}\right) \boldsymbol{R}\left(\hat{\boldsymbol{t}}_{0}, \phi\right)$ that means that the operators $\boldsymbol{\Lambda}$ and $\boldsymbol{R}$ commute.

Remark. The Lagrangian description of the internal state of the rod is defined by means of the two field $\{\boldsymbol{u}(S), \phi(S)\}$, so that it has four degrees of freedom.

According to differential geometry the curvature and torsion of the rod axis are:

$$
\begin{array}{lcc}
\chi_{r n} & \frac{1}{R_{n}} & \frac{1}{\|\boldsymbol{t}\|} \frac{d \hat{\boldsymbol{v}}}{d S} \cdot \hat{\boldsymbol{t}} \\
\chi_{r v} & \frac{1}{R_{v}} & \frac{1}{\|\boldsymbol{t}\|} \frac{d \hat{\boldsymbol{n}}}{d S} \cdot \hat{\boldsymbol{t}} \\
\chi_{t} & \frac{1}{\tau} & \frac{1}{\|\boldsymbol{t}\|} \frac{d \hat{\boldsymbol{n}}}{d S} \cdot \hat{\boldsymbol{v}}
\end{array}
$$

The first two are the (reduced) bending curvatures, and are equal to the components of the vector $\frac{1}{\|t\|} \hat{\boldsymbol{t}} \times \frac{d \hat{t}}{d S}$, while the third is the tor sional curvature, or twist. The name "reduced" has been added for consistency with later definitions. In the initial configuration the torsional curvature is $\chi_{t 0} \quad \frac{d \hat{\boldsymbol{n}}_{0}}{d S} \hat{\boldsymbol{v}}_{0} \quad \frac{d \phi_{0}}{d S}$, since the intermediate frame $\left(\hat{\boldsymbol{n}}_{0}^{b}, \hat{\boldsymbol{v}}_{0}^{b}\right)$ is geodetic by construction. On the contrary, in the deformed configuration, the torsional curvature depends both from $\frac{d \phi}{d S}$ and from the deformation of the centroid axis, as appears from an easy calculation,

$\chi_{t} \quad \frac{1}{\|\boldsymbol{t}\|} \boldsymbol{R}^{T} \frac{d \boldsymbol{R}}{d S} \hat{\boldsymbol{n}}^{b} \cdot \hat{\boldsymbol{v}}^{b}+\frac{1}{\|\boldsymbol{t}\|} \boldsymbol{\Lambda}^{T} \frac{d \boldsymbol{\Lambda}}{d S} \hat{\boldsymbol{n}}_{0} \cdot \hat{\boldsymbol{v}}_{0}+\frac{1}{\|\boldsymbol{t}\|} \frac{d \hat{\boldsymbol{n}}_{0}}{d S} \cdot \hat{\boldsymbol{v}}_{0}$,

the latter term being the initial twist referred, however, to the de formed arc length.

\section{Tangent kinematics}

In order to obtain the expression of the mechanical power for the rod, we need to evaluate the velocity of the deformed configu ration. In this section, first the velocity of the intrinsic triad is de rived, then we use this result for determining an expression for the velocity of deformation. Since we use a material description, the velocity is formally obtained performing the partial time derivative of the pull back of the relevant objects on the material frame (see Simo and Fox [23], p. 287).

\subsection{Velocity of the intrinsic triad}

The evolution of the intrinsic frame can be derived in terms of the rotation vector $\boldsymbol{\omega} \quad \dot{\phi} \hat{\boldsymbol{t}}+\omega_{n} \hat{\boldsymbol{n}}+\omega_{v} \hat{\boldsymbol{v}}$ as

$\begin{array}{llcl}\dot{\hat{\boldsymbol{t}}} & \boldsymbol{\omega} \times \hat{\boldsymbol{t}} & \omega_{v} \hat{\boldsymbol{n}} & \omega_{n} \hat{\boldsymbol{v}} \\ \dot{\hat{\boldsymbol{n}}} & \boldsymbol{\omega} \times \hat{\boldsymbol{n}} & \omega_{v} \hat{\boldsymbol{t}} & +\dot{\phi} \hat{\boldsymbol{v}} \\ \dot{\hat{\boldsymbol{v}}} & \boldsymbol{\omega} \times \hat{\boldsymbol{v}} & \omega_{n} \hat{\boldsymbol{t}} & \dot{\phi} \hat{\boldsymbol{n}}\end{array}$

From the previous definition it follows that

$$
\begin{array}{lccc}
\dot{\phi} & \dot{\hat{\boldsymbol{n}}} \cdot \hat{\boldsymbol{v}} & \dot{\hat{\boldsymbol{v}}} \cdot \hat{\boldsymbol{n}} \\
\omega_{n} & \dot{\hat{\boldsymbol{t}}} \cdot \hat{\boldsymbol{v}} & \dot{\hat{\boldsymbol{v}}} \cdot \hat{\boldsymbol{t}} & \frac{1}{\|\boldsymbol{t}\|} \frac{d \dot{\boldsymbol{u}}}{d S} \cdot \hat{\boldsymbol{v}} \\
\omega_{v} & \dot{\hat{\boldsymbol{t}}} \cdot \hat{\boldsymbol{n}} & \dot{\hat{\boldsymbol{n}}} \cdot \hat{\boldsymbol{t}} & \frac{1}{\|\boldsymbol{t}\|} \frac{d \dot{\boldsymbol{u}}}{d S} \cdot \hat{\boldsymbol{n}} .
\end{array}
$$

The first component of the velocity of rotation is indeed the velocity of the twist angle introduced in Eq. (11). As a matter of fact, the re sults (16) can be equivalently obtained by means of the rotation operators, i.e.

\section{$\dot{\hat{\boldsymbol{n}}} \quad \dot{\boldsymbol{Q}} \hat{\boldsymbol{n}}_{0} \quad \dot{\boldsymbol{Q} Q}{ }^{1} \hat{\boldsymbol{n}} \quad \dot{\boldsymbol{R}} \boldsymbol{R}^{T} \hat{\boldsymbol{n}}+\boldsymbol{R} \dot{\boldsymbol{\Lambda}} \boldsymbol{\Lambda}^{T} \boldsymbol{R}^{T} \hat{\boldsymbol{n}}$}

It follows that

$\dot{\hat{\boldsymbol{n}}} \cdot \hat{\boldsymbol{v}} \quad \dot{\boldsymbol{R}} \boldsymbol{R}^{T} \hat{\boldsymbol{n}} \cdot \hat{\boldsymbol{v}}+\dot{\boldsymbol{\Lambda}} \boldsymbol{\Lambda}^{T} \hat{\boldsymbol{n}}^{b} \cdot \hat{\boldsymbol{v}}^{b} \quad \dot{\phi}$

It can be proved that the first term is $\dot{\phi}$, while the second term van ishes, as can be easily understood recalling the definition of the intermediate frame $\left(\hat{\boldsymbol{n}}^{b}, \hat{\boldsymbol{v}}^{b}\right)$.

The derivative along the current arc length of the velocity of rotation $\omega$ is

$\dot{\chi} \quad \frac{1}{\|\boldsymbol{t}\|} \frac{d \omega}{d S} \quad \dot{\chi}_{t} \hat{\boldsymbol{t}}+\dot{\chi}_{n} \hat{\boldsymbol{n}}+\dot{\chi}_{v} \hat{\boldsymbol{v}}$

where the torsional $\left(\dot{\chi}_{t}\right)$ and bending $\left(\dot{\chi}_{n}, \dot{\chi}_{v}\right)$ velocities of curvature are given by the following expressions

$$
\begin{array}{ll}
\dot{\chi}_{t} \quad \frac{1}{\|\boldsymbol{t}\|} \frac{d \dot{\phi}}{d S} \frac{\omega_{n}}{R_{v}}+\frac{\omega_{v}}{R_{n}} \\
\dot{\chi}_{n} \quad \frac{\dot{\phi}}{R_{v}}+\frac{1}{\|\boldsymbol{t}\|} \frac{d \omega_{n}}{d S} \frac{\omega_{v}}{\tau} \\
\dot{\chi}_{v} \quad \frac{\dot{\phi}}{R_{n}}+\frac{\omega_{n}}{\tau}+\frac{1}{\|\boldsymbol{t}\|} \frac{d \omega_{v}}{d S} .
\end{array}
$$

In the bending velocity of curvature appear the derivatives of the components of the velocity, that are related to the second covariant derivative of the displacement vector. The first and the second covariant line gradients of the vector $\dot{\boldsymbol{u}}$ are given by

$$
\begin{array}{ll}
\operatorname{Grad}(\dot{\boldsymbol{u}}) & \frac{d \dot{\boldsymbol{u}}}{d S} \otimes \boldsymbol{t}^{\natural} \\
\operatorname{Grad}^{2}(\dot{\boldsymbol{u}}) & \frac{d}{d S}\left(\frac{d \dot{\boldsymbol{u}}}{d S} \otimes \boldsymbol{t}^{\natural}\right) \otimes \boldsymbol{t}^{\natural} \\
& \frac{d}{d S}\left(\frac{d \dot{\boldsymbol{u}}}{d S}\right) \otimes \boldsymbol{t}^{\natural} \otimes \boldsymbol{t}^{\natural}+\frac{d \dot{\boldsymbol{u}}}{d S} \otimes \frac{d \boldsymbol{t}^{\natural}}{d S} \otimes \boldsymbol{t}^{\natural}
\end{array}
$$


having indicated by $\boldsymbol{t}^{\natural}$ the contravariant tangent vector, $\boldsymbol{t}^{\natural} \frac{\boldsymbol{t}}{\|\boldsymbol{t}\|^{2}}$.

The only significant covariant component is along the $S$ coordi nate, so that, since $\boldsymbol{t}^{\natural} \quad \boldsymbol{t} \quad 1, \frac{d \boldsymbol{t}^{\natural}}{d S} \boldsymbol{t} \quad \frac{d \boldsymbol{t}}{d S} \boldsymbol{t}^{\natural}$, the last expression can be manipulated as follows:

$$
\begin{aligned}
\operatorname{Grad}^{2}(\dot{\boldsymbol{u}}) \quad & \frac{d}{d S}\left(\frac{d \dot{\boldsymbol{u}}}{d S}\right) \otimes \boldsymbol{t}^{\natural} \otimes \boldsymbol{t}^{\natural}+\frac{d \dot{\boldsymbol{u}}}{d S}\left(\frac{d \boldsymbol{t}^{\natural}}{d S} \cdot \boldsymbol{t}\right) \otimes \boldsymbol{t}^{\natural} \otimes \boldsymbol{t}^{\natural} \\
& {\left[\frac{d}{d S}\left(\frac{d \dot{\boldsymbol{u}}}{d S}\right) \quad \frac{d \dot{\boldsymbol{u}}}{d S}\left(\frac{d \boldsymbol{t}}{d S} \cdot \boldsymbol{t}^{\natural}\right)\right] \otimes \boldsymbol{t}^{\natural} \otimes \boldsymbol{t}^{\natural} } \\
& \|\boldsymbol{t}\| \frac{d}{d S}\left(\frac{1}{\|\boldsymbol{t}\|} \frac{d \dot{\boldsymbol{u}}}{d S}\right) \otimes \boldsymbol{t}^{\natural} \otimes \boldsymbol{t}^{\natural} \\
& \frac{d^{2} \dot{\boldsymbol{u}}}{d S^{2}} \otimes \boldsymbol{t}^{\natural} \otimes \boldsymbol{t}^{\natural}
\end{aligned}
$$

Performing the derivatives indicated in the third line of (23) we obtain

$$
\begin{array}{ll}
\frac{d^{2} \dot{\boldsymbol{u}}}{d S^{2}} \cdot \hat{\boldsymbol{n}} & \|\boldsymbol{t}\| \frac{d \omega_{v}}{d S}+\frac{\|\boldsymbol{t}\|^{2}}{\tau} \omega_{n}+\dot{\boldsymbol{t}} \cdot \boldsymbol{t} \frac{1}{R_{v}} \\
\frac{d^{2} \dot{\boldsymbol{u}}}{d S^{2}} \cdot \hat{\boldsymbol{v}} & \|\boldsymbol{t}\| \frac{d \omega_{n}}{d S}+\frac{\|\boldsymbol{t}\|^{2}}{\tau} \omega_{v} \quad \dot{\boldsymbol{t}} \cdot \boldsymbol{t} \frac{1}{R_{n}} .
\end{array}
$$

Comparing Eqs. (24) and (21) it is found an alternative expres sion for the bending velocities of curvature:

$$
\begin{array}{lcllllll}
\dot{\chi}_{n} & \frac{1}{\|\boldsymbol{t}\|^{2}} \frac{d^{2} \dot{\boldsymbol{u}}}{d S^{2}} \cdot \hat{\boldsymbol{v}} & \frac{1}{\|\boldsymbol{t}\|^{2}} \frac{\dot{\boldsymbol{t}} \cdot \boldsymbol{t}}{R_{n}}+\frac{\dot{\phi}}{R_{v}} & \frac{\dot{\chi}_{r n}}{\|\boldsymbol{t}\|^{2}} & \frac{1}{\|\boldsymbol{t}\|^{2}} \frac{\dot{\boldsymbol{t}} \cdot \boldsymbol{t}}{R_{n}} \\
\dot{\chi}_{v} & \frac{1}{\|\boldsymbol{t}\|^{2}} \frac{d^{2} \dot{\boldsymbol{u}}}{d S^{2}} \cdot \hat{\boldsymbol{n}} & \frac{1}{\|\boldsymbol{t}\|^{2}} \frac{\dot{\boldsymbol{t}} \cdot \boldsymbol{t}}{R_{v}} & \frac{\dot{\phi}}{R_{n}} & \frac{\dot{\chi}_{v v}}{\|\boldsymbol{t}\|^{2}} & \frac{1}{\|\boldsymbol{t}\|^{2}} \frac{\dot{\boldsymbol{t}} \cdot \boldsymbol{t}}{R_{v}}
\end{array}
$$

where the definition of the reduced velocities of curvature $\dot{\chi}_{r}$ have been introduced.

\subsection{The kinematic tangent operator for a Kirchhoff Love rod model}

Introducing the Lagrangian coordinates $\theta^{n}, \theta^{v}$ along the axes $\hat{\boldsymbol{n}}, \hat{\boldsymbol{v}}$ of the cross section, the position vector of a generic point of the rod in the generic configuration is given by

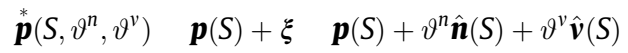

$$
\begin{aligned}
& \boldsymbol{\xi} \quad \vartheta^{n} \hat{\boldsymbol{n}}(S)+\vartheta^{v} \hat{\boldsymbol{v}}(S) .
\end{aligned}
$$

Differentiating Eq. (26) the tangent vectors that span the tan gent space $T_{\boldsymbol{p}} \mathcal{B}_{t}$ at the point $\stackrel{*}{\boldsymbol{p}}$ are

$$
* * \frac{{ }^{*} \boldsymbol{p}}{\partial S} \quad \frac{\partial \boldsymbol{p}}{\partial S}+\vartheta^{v} \frac{\partial \hat{\boldsymbol{v}}}{\partial S}+\vartheta^{n} \frac{\partial \hat{\boldsymbol{n}}}{\partial S}, \quad \stackrel{*}{\boldsymbol{n}}: \frac{\partial \boldsymbol{*}}{\partial \vartheta^{n}} \quad \hat{\boldsymbol{n}}, \quad \stackrel{*}{\boldsymbol{v}}: \frac{\partial \hat{\boldsymbol{p}}}{\partial \vartheta^{v}} \hat{\boldsymbol{v}}
$$

By means of Eq. (27) it is defined the push forward operator from the centroid line of the rod to the generic fibre,

$$
\boldsymbol{z} \quad \stackrel{\boldsymbol{g}}{\alpha}_{\alpha} \otimes \mathbf{g}^{\alpha}, \stackrel{\boldsymbol{g}}{\alpha}_{\alpha} \quad\{\hat{\boldsymbol{t}}, \hat{\boldsymbol{n}}, \hat{\boldsymbol{v}}\}, \mathbf{g}_{\alpha} \quad\{\boldsymbol{t}, \hat{\boldsymbol{n}}, \hat{\boldsymbol{v}}\}, \mathbf{g}^{\alpha} \quad\left\{\boldsymbol{t}^{\natural}, \hat{\boldsymbol{n}}, \hat{\boldsymbol{v}}\right\}
$$

where the index ${ }^{\natural}$ indicates contravariant base vectors defined by

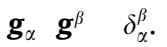

The gradient of deformation from the reference configuration of the axis to its current configuration is $\boldsymbol{F} \mathbf{g}_{\alpha} \otimes \mathbf{g}_{0}^{\alpha}$ $\boldsymbol{t} \otimes \hat{\boldsymbol{t}}_{0}+\hat{\boldsymbol{n}} \otimes \hat{\boldsymbol{n}}_{0}+\hat{\boldsymbol{v}} \otimes \hat{\boldsymbol{v}}_{0}$ (see Fig. 2)

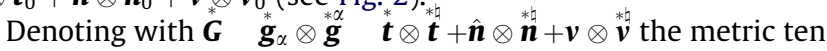
sor at the generic fibre, its pull back on the centroid axis of the ref erence configuration is $\psi^{*}(\boldsymbol{G}) \quad \boldsymbol{F}^{T} \boldsymbol{z}^{T}{ }^{*} \boldsymbol{z} \boldsymbol{F}$, whose components on the material frame $\hat{\boldsymbol{t}}_{0}, \hat{\boldsymbol{n}}_{0}, \hat{\boldsymbol{v}}_{0}$ are

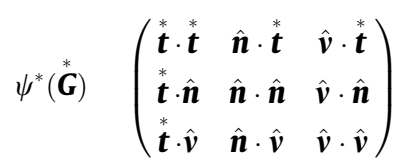

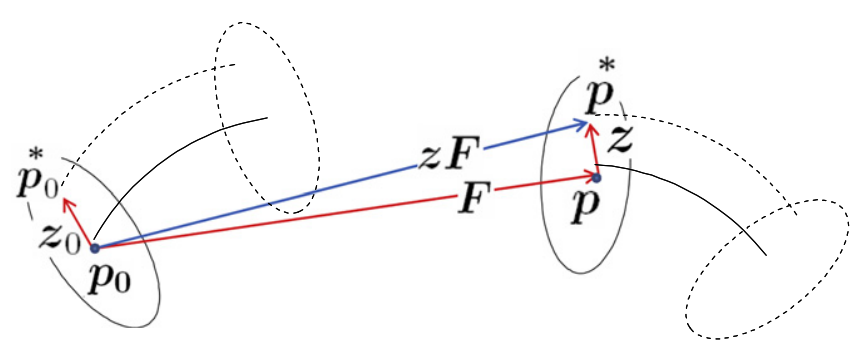

Fig. 2. Transformations from the reference to the current configuration and from the tangent space to the centroid axes to the tangent space to the generic fibre.

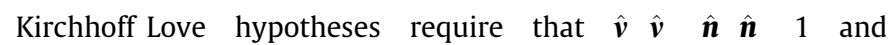
$\hat{\boldsymbol{v}} \hat{\boldsymbol{n}} 0$, then the metric tensor (29) becomes

$\psi^{*}(\boldsymbol{G}) \quad\left(\begin{array}{ccc}* & * * \\ \boldsymbol{t} \cdot \boldsymbol{t} & \hat{\boldsymbol{n}} \cdot{ }^{*} & \hat{\boldsymbol{v}} \cdot \boldsymbol{t} \\ \boldsymbol{t} \cdot \hat{\boldsymbol{n}} & 1 & 0 \\ * \\ \boldsymbol{t} \cdot \hat{\boldsymbol{v}} & 0 & 1\end{array}\right)$

The Green Lagrange deformation tensor is thus obtained as

$\boldsymbol{E} \quad \frac{1}{2}\left(\psi^{*}(\stackrel{*}{\boldsymbol{G}}) \quad \boldsymbol{z}_{0}^{T} \stackrel{*}{\mathbf{G}_{0}} \boldsymbol{z}_{0}\right)$

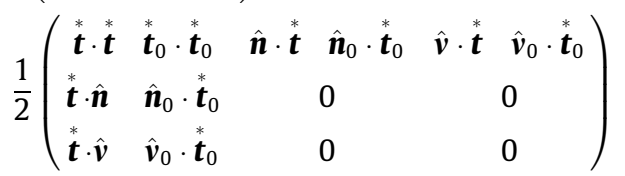

According to the results of Section 3 and disregarding terms of order higher than 1 in $\vartheta^{n}, \vartheta^{v}$ we find

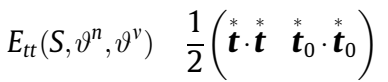

$$
\begin{aligned}
& \frac{1}{2}\left(\boldsymbol{t} \cdot \boldsymbol{t} \quad \boldsymbol{t}_{0} \cdot \boldsymbol{t}_{0}\right)+\vartheta^{n}\left(\frac{d \hat{\boldsymbol{n}}}{d S} \cdot \boldsymbol{t} \quad \frac{d \hat{\boldsymbol{n}}_{0}}{d S} \cdot \hat{\boldsymbol{t}}_{0}\right) \\
& +\vartheta^{v}\left(\frac{d \hat{\boldsymbol{v}}}{d S} \cdot \boldsymbol{t} \frac{d \hat{\boldsymbol{v}}_{0}}{d S} \cdot \hat{\boldsymbol{t}}_{0}\right)+\text { higher order terms } \\
& \frac{1}{2}\left(\boldsymbol{t} \cdot \boldsymbol{t} \quad \boldsymbol{t}_{0} \cdot \boldsymbol{t}_{0}\right) \quad \vartheta^{n}\left(\begin{array}{ll}
\frac{\|\boldsymbol{t}\|^{2}}{R_{v}} & \frac{1}{R_{v 0}}
\end{array}\right)+\vartheta^{v}\left(\begin{array}{ll}
\frac{\|\boldsymbol{t}\|^{2}}{R_{n}} & \frac{1}{R_{n 0}}
\end{array}\right)
\end{aligned}
$$

the first term is the extensional deformation of the axis. Analo gously we have

$$
\begin{array}{llll}
E_{t n} & \frac{1}{2} \hat{\boldsymbol{n}} \cdot \boldsymbol{t}^{*} & \hat{\boldsymbol{n}}_{0} \cdot \boldsymbol{t}_{0} & \frac{1}{2} \vartheta^{v}\left(\begin{array}{lll}
\frac{\|\boldsymbol{t}\|}{\tau} & \frac{1}{\tau_{0}}
\end{array}\right) \\
E_{t v} & \frac{1}{2} \hat{\boldsymbol{v}} \cdot \boldsymbol{t} & \hat{\boldsymbol{v}}_{0} \cdot \boldsymbol{t}_{0} & \frac{1}{2} \vartheta^{n}\left(\begin{array}{ll}
\frac{\|\boldsymbol{t}\|}{\tau} & \frac{1}{\tau_{0}}
\end{array}\right) .
\end{array}
$$

The velocity of deformation tensor at the generic quota is:

d $\operatorname{sym}\left(\dot{\bar{z} \boldsymbol{F}}(\boldsymbol{z F})^{1}\right)$,

its pull back on the centroid axis of the current configuration is

$$
\text { d } \quad \boldsymbol{z}^{T} \stackrel{*}{\mathbf{d} z},
$$

and the pull back on the centroid axis of the reference configuration is

$\boldsymbol{D} \quad \dot{\boldsymbol{E}} \quad \operatorname{sym}\left((\boldsymbol{z} \boldsymbol{F})^{T} \dot{\overline{\boldsymbol{z}} \boldsymbol{F}}\right) \quad D_{\alpha \beta} \mathbf{g}_{0}^{\alpha} \otimes \mathbf{g}_{0}^{\beta}$.

The components of the last tensor on the material frame are

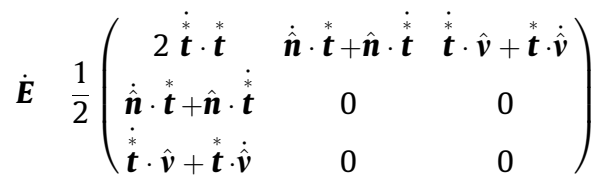


The following sections concern the derivation of the compo nents of the velocity of deformation.

\subsubsection{Shear deformation velocity $\dot{\gamma}$}

The components of the shear deformation velocity are given by the off diagonal terms of tensor (37)

$$
\dot{\gamma}_{\hat{n} t} \quad \dot{\hat{\boldsymbol{n}}} \cdot \boldsymbol{t}+\hat{\boldsymbol{n}} \cdot \boldsymbol{*} \quad \dot{\gamma}_{\hat{v} t}^{*} \quad \dot{*} \cdot \hat{\boldsymbol{v}}+\stackrel{*}{\boldsymbol{t}} \cdot \dot{\hat{\boldsymbol{v}}}
$$

Using Eqs. (27) and (16) we have

$$
\begin{array}{ll}
\dot{\gamma}_{\hat{n} t} \quad(\dot{\boldsymbol{t}} \cdot \hat{\boldsymbol{n}}+\boldsymbol{t} \cdot \dot{\hat{\boldsymbol{n}}})+\vartheta^{n}\left(\frac{d \dot{\hat{\boldsymbol{n}}}}{d S} \cdot \hat{\boldsymbol{n}}+\frac{d \hat{\boldsymbol{n}}}{d S} \cdot \dot{\hat{\boldsymbol{n}}}\right)+\vartheta^{v}\left(\frac{d \dot{\hat{\boldsymbol{v}}}}{d S} \cdot \hat{\boldsymbol{n}}+\frac{d \hat{\boldsymbol{v}}}{d S} \cdot \dot{\hat{\boldsymbol{n}}}\right) \\
\dot{\gamma}_{\hat{v} t} \quad(\dot{\boldsymbol{t}} \cdot \hat{\boldsymbol{v}}+\boldsymbol{t} \cdot \dot{\hat{\boldsymbol{v}}})+\vartheta^{n}\left(\frac{d \dot{\hat{\boldsymbol{n}}}}{d S} \cdot \hat{\boldsymbol{v}}+\frac{d \hat{\boldsymbol{n}}}{d S} \cdot \dot{\hat{\boldsymbol{v}}}\right)+\vartheta^{v}\left(\frac{d \dot{\hat{\boldsymbol{v}}}}{d S} \cdot \hat{\boldsymbol{v}}+\frac{d \hat{\boldsymbol{v}}}{d S} \cdot \dot{\hat{\boldsymbol{v}}}\right) .
\end{array}
$$

The time derivative of Kirchhoff Love's constraints $\begin{array}{llllllll}\boldsymbol{t} & \hat{\boldsymbol{n}} & 0, \boldsymbol{t} & \hat{\boldsymbol{v}} & 0, \text { and the equalities } \frac{d \hat{\boldsymbol{n}}}{d S} \hat{\boldsymbol{n}} & 0, \frac{d \hat{v}}{d S} \hat{\boldsymbol{v}} \quad, \hat{\boldsymbol{n}} & \hat{\boldsymbol{v}} & 0,\end{array}$ reduce (39) to

$$
\begin{aligned}
& \dot{\gamma}_{\hat{n}^{*} t} \quad \vartheta^{v} \dot{\gamma} \quad \dot{\gamma}_{\hat{v} t} \quad \vartheta^{n} \dot{\gamma} \\
& \dot{\gamma} \quad\left(\frac{d \dot{\hat{\boldsymbol{n}}}}{d S} \cdot \hat{\boldsymbol{v}}+\frac{d \hat{\boldsymbol{n}}}{d S} \cdot \dot{\hat{\boldsymbol{v}}}\right) .
\end{aligned}
$$

Using the expressions for the velocities of rotation of the refer ence frame it is readily found

$$
\begin{aligned}
\dot{\gamma} \quad & \frac{d}{d S}(\boldsymbol{\omega} \times \hat{\boldsymbol{n}}) \cdot \hat{\boldsymbol{v}}+\frac{d \hat{\boldsymbol{n}}}{d S} \cdot(\boldsymbol{\omega} \times \hat{\boldsymbol{v}}) \\
& \hat{\boldsymbol{n}} \times \hat{\boldsymbol{v}} \cdot \frac{d \omega}{d S}+\hat{\boldsymbol{v}} \times \boldsymbol{\omega} \cdot \frac{d \hat{\boldsymbol{n}}}{d S}+\frac{d \hat{\boldsymbol{n}}}{d S} \cdot(\boldsymbol{\omega} \times \hat{\boldsymbol{v}}) \\
& \frac{d \boldsymbol{\omega}}{d S} \cdot \hat{\boldsymbol{t}} \quad\|\boldsymbol{t}\| \dot{\chi}_{t}
\end{aligned}
$$

The result (21), definitions (17) and the identity

$\hat{\boldsymbol{t}} \times \frac{d \hat{\boldsymbol{t}}}{d S} \quad\|\boldsymbol{t}\|\left(\frac{\hat{\boldsymbol{n}}}{R_{n}}+\frac{\hat{\boldsymbol{v}}}{R_{v}}\right)$

allow to get a representation of $\dot{\gamma}$ in terms of the Lagrangian gener alized velocity vector $\dot{\boldsymbol{q}} \quad\{\dot{\boldsymbol{u}}, \dot{\phi}\}$ :

$\dot{\gamma} \frac{1}{\|\boldsymbol{t}\|}\left(\hat{\boldsymbol{t}} \times \frac{d \hat{\boldsymbol{t}}}{d S}\right) \cdot \frac{d \dot{\boldsymbol{u}}}{d S}+\frac{d \dot{\phi}}{d S}$.

\subsubsection{Membrane and curvature velocities of deformation}

The axial velocity of deformation, using (27), is given by

$$
\begin{aligned}
& \stackrel{*}{t} \cdot \boldsymbol{t}^{*} \quad \dot{\boldsymbol{t}} \cdot \boldsymbol{t}+\vartheta^{v}\left(\frac{d \dot{\hat{\boldsymbol{v}}}}{d S} \cdot \boldsymbol{t}+\frac{d \hat{\boldsymbol{v}}}{d S} \cdot \dot{\boldsymbol{t}}\right)+\vartheta^{n}\left(\frac{d \dot{\hat{\boldsymbol{n}}}}{d S} \cdot \boldsymbol{t}+\frac{d \hat{\boldsymbol{n}}}{d S} \cdot \dot{\boldsymbol{t}}\right) \\
& \quad+\left(\vartheta^{v}\right)^{2} \frac{d \dot{\hat{\boldsymbol{v}}}}{d S} \cdot \frac{d \hat{\boldsymbol{v}}}{d S}+\vartheta^{v} \vartheta^{n}\left(\frac{d \dot{\hat{\boldsymbol{v}}}}{d S} \cdot \frac{d \hat{\boldsymbol{n}}}{d S}+\frac{d \hat{\boldsymbol{v}}}{d S} \cdot \frac{d \dot{\hat{\boldsymbol{n}}}}{d S}\right)+\left(\vartheta^{n}\right)^{2} \frac{d \dot{\hat{\boldsymbol{n}}}}{d S} \cdot \frac{d \hat{\boldsymbol{n}}}{d S} .
\end{aligned}
$$

Proceeding as above, we have:

$$
\begin{aligned}
\stackrel{*}{\boldsymbol{t}} \cdot * \boldsymbol{t} & \dot{\boldsymbol{t}} \cdot \boldsymbol{t}+\vartheta^{n}\left(\boldsymbol{t} \cdot \frac{d}{d S}(\boldsymbol{\omega} \times \hat{\boldsymbol{n}})+\dot{\boldsymbol{t}} \cdot \frac{d \hat{\boldsymbol{n}}}{d S}\right) \\
& +\vartheta^{v}\left(\boldsymbol{t} \cdot \frac{d}{d S}(\boldsymbol{\omega} \times \hat{\boldsymbol{v}})+\dot{\boldsymbol{t}} \cdot \frac{d \hat{\boldsymbol{v}}}{d S}\right) \\
& +\vartheta^{n} \vartheta^{v}\left(\frac{d \hat{\boldsymbol{n}}}{d S} \cdot \frac{d}{d S}(\boldsymbol{\omega} \times \hat{\boldsymbol{v}})+\frac{d \hat{\boldsymbol{v}}}{d S} \cdot \frac{d}{d S}(\boldsymbol{\omega} \times \hat{\boldsymbol{n}})\right) \\
& +\left(\vartheta^{n}\right)^{2}\left(\frac{d \hat{\boldsymbol{n}}}{d S} \cdot \frac{d}{d S}(\boldsymbol{\omega} \times \hat{\boldsymbol{n}})\right)+\left(\vartheta^{v}\right)^{2}\left(\frac{d \hat{\boldsymbol{v}}}{d S} \cdot \frac{d}{d S}(\boldsymbol{\omega} \times \hat{\boldsymbol{v}})\right)
\end{aligned}
$$

The term in the first parenthesis can be treated as follows: $\boldsymbol{t} \cdot \frac{d \boldsymbol{\omega}}{d S} \times \hat{\boldsymbol{n}}+\boldsymbol{t} \cdot \boldsymbol{\omega} \times \frac{d \hat{\boldsymbol{n}}}{d S}+\dot{\boldsymbol{t}} \cdot \frac{d \hat{\boldsymbol{n}}}{d S} \quad \frac{d \boldsymbol{\omega}}{d S} \cdot \hat{\boldsymbol{v}}\|\boldsymbol{t}\|+\|\boldsymbol{t}\| \hat{\boldsymbol{t}} \times \boldsymbol{\omega} \cdot \frac{d \hat{\boldsymbol{n}}}{d S}$

$$
\begin{array}{r}
+\|\boldsymbol{t}\| \dot{\hat{\boldsymbol{t}}} \cdot \frac{d \hat{\boldsymbol{n}}}{d S}+\frac{\dot{\| \boldsymbol{t}} \| \hat{\boldsymbol{t}}}{d S} \frac{d \hat{\boldsymbol{n}}}{d S} \\
\frac{d \omega}{d S} \cdot \hat{\boldsymbol{v}}\|\boldsymbol{t}\| \quad \dot{\boldsymbol{t}} \cdot \boldsymbol{t} \frac{1}{R_{v}}
\end{array}
$$

Analogous transformations lead to

$$
\begin{array}{cc}
\dot{\boldsymbol{t}}^{*} \cdot \boldsymbol{t} & \dot{\boldsymbol{t}} \cdot \boldsymbol{t}+\vartheta^{n}\left(\frac{d \boldsymbol{\omega}}{d S} \cdot \hat{\boldsymbol{v}}\|\boldsymbol{t}\| \quad \dot{\boldsymbol{t}} \cdot \boldsymbol{t} \frac{1}{R_{v}}\right)+\vartheta^{v}\left(\frac{d \boldsymbol{\omega}}{d S} \cdot \hat{\boldsymbol{n}}\|\boldsymbol{t}\|+\dot{\boldsymbol{t}} \cdot \boldsymbol{t} \frac{1}{R_{n}}\right) \\
& +\vartheta^{n} \vartheta^{v} \cdot\left(\frac{d \boldsymbol{\omega}}{d S}\left(\hat{\boldsymbol{v}} \times \frac{d \hat{\boldsymbol{n}}}{d S}+\hat{\boldsymbol{n}} \times \frac{d \hat{\boldsymbol{v}}}{d S}\right)\right)+\left(\vartheta^{n}\right)^{2}\left(\frac{d \boldsymbol{\omega}}{d S} \cdot \hat{\boldsymbol{n}} \times \frac{d \hat{\boldsymbol{n}}}{d S}\right) \\
& +\left(\vartheta^{v}\right)^{2}\left(\frac{d \boldsymbol{\omega}}{d S} \cdot \hat{\boldsymbol{v}} \times \frac{d \hat{\boldsymbol{v}}}{d S}\right)
\end{array}
$$

Accounting for the equalities

$$
\begin{array}{cccc}
\hat{\boldsymbol{v}} \times \frac{d \hat{\boldsymbol{n}}}{d S}+\hat{\boldsymbol{n}} \times \frac{d \hat{\boldsymbol{v}}}{d S} \quad \hat{\boldsymbol{n}} \frac{\|\boldsymbol{t}\|}{R_{v}} \quad \hat{\boldsymbol{v}} \frac{\|\boldsymbol{t}\|}{R_{n}} \\
\frac{1}{\|\boldsymbol{t}\|} \frac{d \hat{\boldsymbol{t}}}{d S} \quad \frac{\hat{\boldsymbol{n}}}{R_{v}} \quad \frac{\hat{\boldsymbol{v}}}{R_{n}}
\end{array}
$$

the result (47) can be cast in the form:

$\stackrel{*}{\boldsymbol{t}} \cdot \stackrel{*}{\boldsymbol{t}} \quad \stackrel{\dot{*}}{\varepsilon}+\dot{\boldsymbol{\chi}}^{\perp} \cdot \boldsymbol{\xi}\|\boldsymbol{t}\|^{2} \quad[\boldsymbol{\xi} \otimes \xi] \dot{\boldsymbol{\chi}}^{\perp} \cdot \frac{d \hat{\boldsymbol{t}}}{d S}+\left(\vartheta^{n^{2}}+\vartheta^{v^{2}}\right) \dot{\boldsymbol{\chi}} t \frac{\|\boldsymbol{t}\|}{\tau}$

with the notations

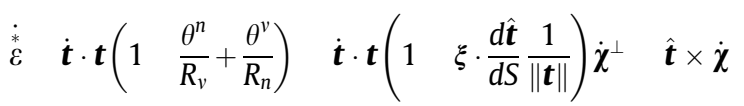$$
\boldsymbol{\xi} \theta^{n} \hat{\boldsymbol{n}}+\theta^{v} \hat{\boldsymbol{v}} .
$$

In this work we disregard the quadratic terms in the section coordinates obtaining the linear representation

$$
\stackrel{\dot{*}}{\boldsymbol{t}} \cdot{ }^{*} \quad \stackrel{*}{\varepsilon}+\boldsymbol{\xi} \cdot \dot{\boldsymbol{\chi}}^{\perp}\|\boldsymbol{t}\| \quad \dot{\boldsymbol{t}} \cdot \boldsymbol{t}\left(1 \quad \frac{\theta^{n}}{R_{v}}+\frac{\theta^{v}}{R_{n}}\right)+\theta^{n}\left(\dot{\chi}_{v}\right)\|\boldsymbol{t}\|^{2}+\theta^{v} \dot{\chi}_{n}\|\boldsymbol{t}\|^{2},
$$

or, substituting the expressions (21) of the velocities of curvatures,

$$
\begin{gathered}
\dot{*} \cdot \boldsymbol{t} \\
\dot{\boldsymbol{t}} \cdot \boldsymbol{t}\left(1 \quad \frac{\theta^{n}}{R_{v}}+\frac{\theta^{v}}{R_{n}}\right)+\theta^{n}\|\boldsymbol{t}\|^{2}\left(\frac{1}{\|\boldsymbol{t}\|} \frac{d \omega_{v}}{d S}+\frac{\dot{\phi}}{R_{n}} \frac{\omega_{n}}{\tau}\right) \\
+\theta^{v}\|\boldsymbol{t}\|^{2}\left(\frac{1}{\|\boldsymbol{t}\|} \frac{d \omega_{n}}{d S}+\frac{\dot{\phi}}{R_{v}} \frac{\omega_{v}}{\tau}\right)
\end{gathered}
$$

The velocities of curvature can be related directly to the dis placement degrees of freedom. Using Eq. (25) and the result (42) the bending curvature vector becomes:

$$
\|\boldsymbol{t}\|^{2} \dot{\chi}^{\perp} \quad \frac{d^{2} \dot{\boldsymbol{u}}}{d S^{2}}+\dot{\boldsymbol{t}} \cdot \boldsymbol{t} \frac{1}{\|\boldsymbol{t}\|} \frac{d \hat{\boldsymbol{t}}}{d S}+\hat{\boldsymbol{t}} \times \frac{d \hat{\boldsymbol{t}}}{d S} \dot{\phi}\|\boldsymbol{t}\|
$$

It is therefore possible to obtain an alternative expression for the axial velocity of deformation, noting that the second addend in Eq. (54) cancels out with the second and third terms of $\stackrel{*}{\varepsilon}$,

$$
\begin{aligned}
& \dot{*} \cdot \stackrel{*}{\boldsymbol{t}} \quad \dot{\varepsilon}+\xi \cdot \dot{\boldsymbol{\chi}}_{\boldsymbol{r}}{ }^{\perp}\|\boldsymbol{t}\|^{2} \\
& \dot{\varepsilon} \quad \dot{\boldsymbol{t}} \cdot \boldsymbol{t} \\
& \|\boldsymbol{t}\|^{2} \dot{\boldsymbol{\chi}}_{\boldsymbol{r}}{ }^{\perp} \quad \frac{d^{2} \dot{\boldsymbol{u}}}{d S^{2}}+\left(\hat{\boldsymbol{t}} \times \frac{d \boldsymbol{t}}{d S}\right)\|\boldsymbol{t}\| \dot{\phi}
\end{aligned}
$$

\section{The equilibrium equation of the Kirchhoff-Love rod}

\subsection{The equilibrium operator}

The equilibrium operator is obtained from the principle of vir tual power. The internal power is 


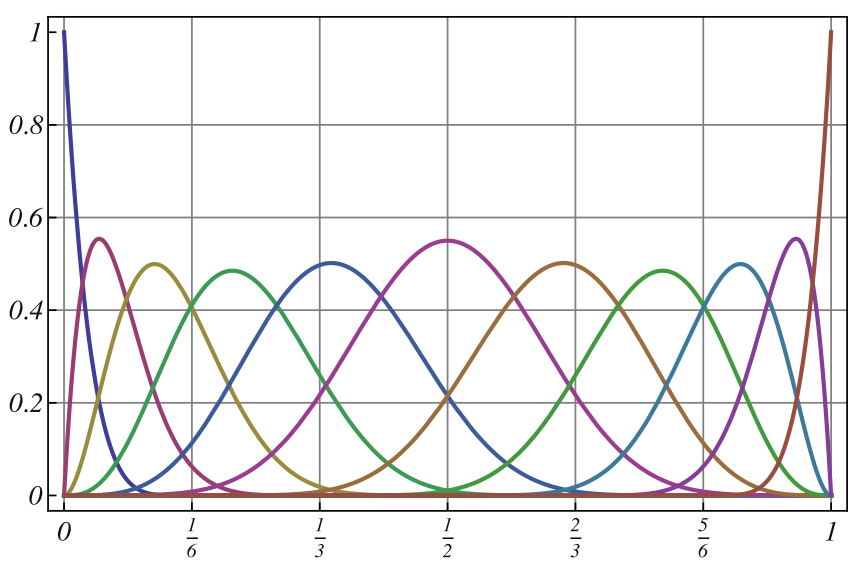

Fig. 3. Quintic B-Spline basis functions with open knot vector $\Xi=\{0,0,0,0,0,0,1 /$ $6,2 / 6,3 / 6,4 / 6,5 / 6,1,1,1,1,1,1\}$.

$P_{\text {int }} \quad \int_{L}^{*}\left(\int_{\mathcal{A}} \stackrel{*}{\Sigma}:^{*} \mathbf{d} d \mathcal{A}\right) d \stackrel{\text { s }}{*}$

According to the beam model, we assume $\stackrel{* n n}{\Sigma} \stackrel{* v v}{\Sigma} \stackrel{* n v}{\Sigma} 0 ; \stackrel{*}{\Sigma}^{*}$ is the Cauchy stress tensor acting at point $\boldsymbol{p}$. It is more convenient to refer to its pull back on the tangent space to the centroid curve,
$\boldsymbol{\Sigma} \quad \Sigma^{\alpha \beta} \mathbf{g}_{\alpha} \otimes \mathbf{g}_{\beta} \operatorname{det}(\boldsymbol{z}) \boldsymbol{z}^{1}{ }^{*} \boldsymbol{\Sigma}^{*} \boldsymbol{z}^{T}$, with $\mathbf{g}_{\alpha}\{\boldsymbol{t}, \hat{\boldsymbol{n}}, \hat{\boldsymbol{v}}\}$, so that the internal power is

$P_{\text {int }} \quad \int_{L}\left(\int_{\mathcal{A}} \boldsymbol{\Sigma}: \boldsymbol{d} d \mathcal{A}\right) d s$

with

$\operatorname{det}(\boldsymbol{z}) \quad\left(1 \quad \frac{\vartheta^{n}}{R_{v}}+\frac{\vartheta^{v}}{R_{n}}\right)$

Alternatively, it is possible to use the representation of the internal power on the reference configuration:

$P_{\text {int }} \int_{L_{0}}\left(\int_{\mathcal{A}} \boldsymbol{S}: \dot{\boldsymbol{E}} d \mathcal{A}\right) d S$,

with $\boldsymbol{S}=S^{\alpha \beta} \mathbf{g}_{0 \alpha} \otimes \mathbf{g}_{0 \beta}$ the second Piola Kirchhoff stress tensor, given by

$\boldsymbol{S} \operatorname{det}(\boldsymbol{z} \boldsymbol{F})(\boldsymbol{z} \boldsymbol{F}){ }^{1} \stackrel{*}{\boldsymbol{\Sigma}}(\boldsymbol{z} \boldsymbol{F})^{T}$

Its components on the reference unitary centroid triads are

$S\left(\begin{array}{ccc}S^{t t} & S^{t n} & S^{t v} \\ S^{n t} & 0 & 0 \\ S^{v t} & 0 & 0\end{array}\right)$

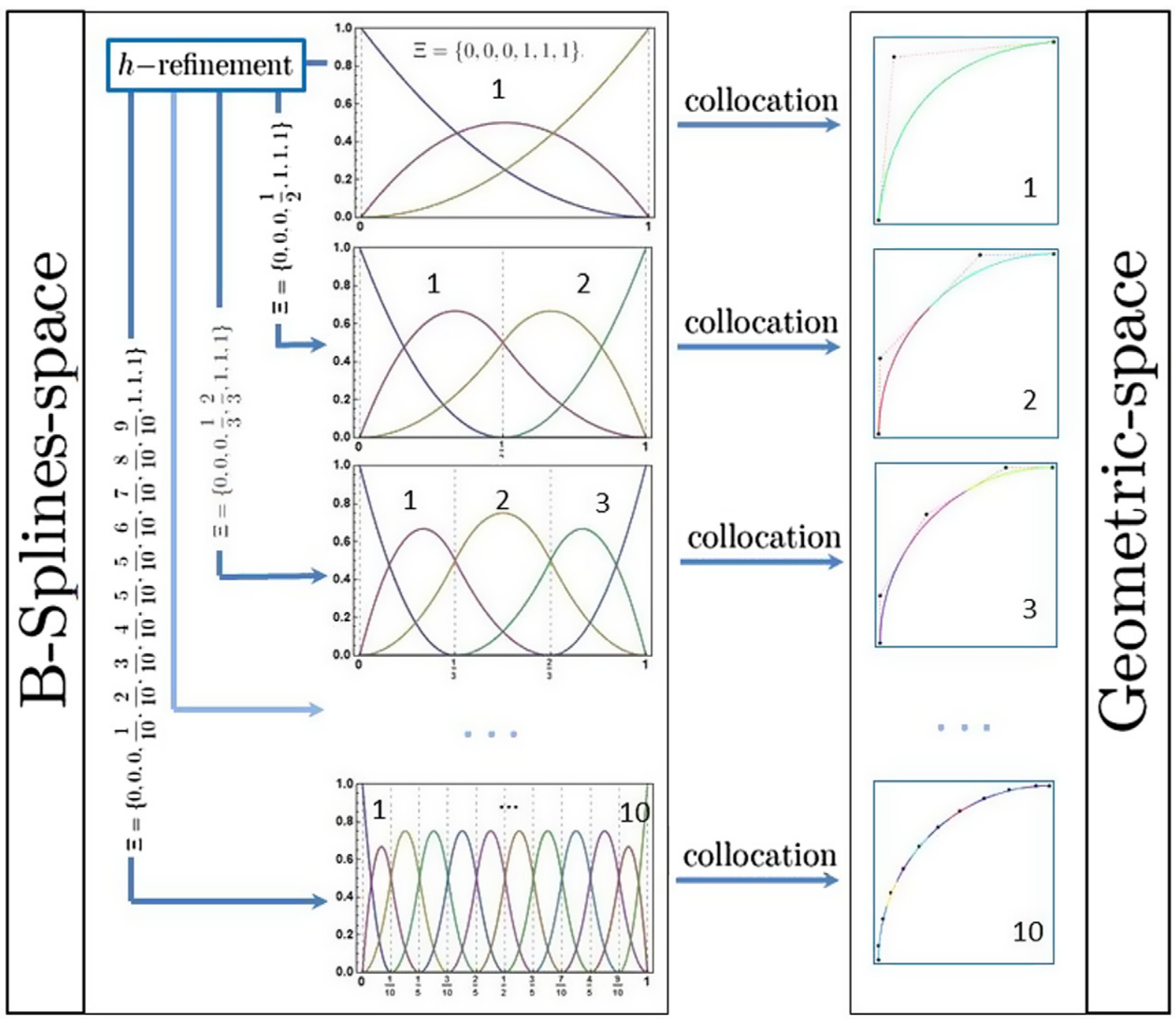

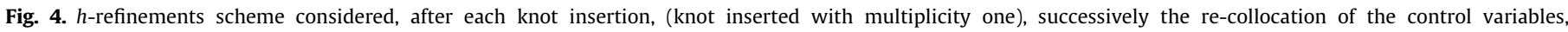
corresponding to the new set of the Greville abscissae, is performed. 
The virtual power identity is therefore (for the sake of simplicity we do not introduce in this expression a symbol for the virtual veloci ties, the meaning being apparent from the context)

$$
\begin{aligned}
\int_{L_{0}}\left(\int_{\mathcal{A}} \boldsymbol{S}: \dot{\boldsymbol{E}} d \mathcal{A}\right) d S & \int_{L_{0}} \boldsymbol{f} \cdot \dot{\boldsymbol{u}} d S+\boldsymbol{F}(0) \cdot \dot{\boldsymbol{u}}(0)+\boldsymbol{F}\left(L_{0}\right) \\
& \cdot \dot{\boldsymbol{u}}\left(L_{0}\right)+\boldsymbol{m}(0) \cdot \boldsymbol{\omega}(0)+\boldsymbol{m}\left(L_{0}\right) \cdot \boldsymbol{\omega}\left(L_{0}\right) .
\end{aligned}
$$

Substituting the components of the velocity of deformation in (62), one has

$$
\begin{aligned}
P_{\text {int }} \quad & \int_{L_{0}}\left(\int_{\mathcal{A}} S^{t t}\left(\dot{\varepsilon} \quad \vartheta^{n} \dot{\chi}_{r v}\|\boldsymbol{t}\|^{2}+\vartheta^{v} \dot{\chi}_{r n}\right)\|\boldsymbol{t}\|^{2}\right. \\
+ & \left.\left(S^{v t} \vartheta^{n} \quad S^{n t} \vartheta^{v}\right) \dot{\chi}_{t}\|\boldsymbol{t}\| d \mathcal{A}\right) d S \\
& \int_{L_{0}}\left(N \dot{\varepsilon}+\boldsymbol{M} \cdot \dot{\boldsymbol{\chi}}_{r}\|\boldsymbol{t}\|^{2}+M_{t} \dot{\chi}_{t}\|\boldsymbol{t}\|\right) d S \\
& \int_{L_{0}}\left(N\left(\frac{d \dot{\boldsymbol{u}}}{d S} \cdot \boldsymbol{t}\right)+M_{n}\left(\frac{d^{2} \dot{\boldsymbol{u}}}{d S^{2}} \cdot \hat{\boldsymbol{v}}+\frac{\|\boldsymbol{t}\|^{2}}{R_{v}} \dot{\phi}\right)\right)+ \\
& M_{v}\left(\frac{d^{2} \dot{\boldsymbol{u}}}{d S^{2}} \cdot \hat{\boldsymbol{n}} \quad \frac{\|\boldsymbol{t}\|^{2}}{R_{n}} \dot{\phi}\right)+M_{t}\left(\frac{d \dot{\phi}}{d S}+\frac{1}{\|\boldsymbol{t}\|} \frac{d \dot{\boldsymbol{u}}}{d S}\left(\hat{\boldsymbol{t}} \times \frac{d \hat{\boldsymbol{t}}}{d S}\right)\right) d S,
\end{aligned}
$$

where the following definitions have been introduced:

$$
\begin{array}{ll}
N & \int_{\mathcal{A}} S^{t t} d \mathcal{A}, \\
\mathbf{M} & \int_{\mathcal{A}} \xi \times\left(S^{t t} \hat{\boldsymbol{t}}\right) d \mathcal{A}, \\
M_{t} & \int_{\mathcal{A}}\left(S^{v t} \vartheta^{n} \quad S^{n t} \vartheta^{v}\right) d \mathcal{A} .
\end{array}
$$

By integrating by parts the terms of the Eq. (63) the equilibrium equations are obtained as follows:

$$
\begin{aligned}
& \frac{d}{d S}\left[N \boldsymbol{t}+\frac{1}{\|\boldsymbol{t}\|} \frac{d}{d S}(\|\boldsymbol{t}\| \boldsymbol{M}) \quad \frac{M_{t}}{\|\boldsymbol{t}\|}\left(\hat{\boldsymbol{t}} \times \frac{d \hat{\boldsymbol{t}}}{d S}\right)\right] \quad \boldsymbol{f} \\
& \frac{d M_{t}}{d S}+\|\boldsymbol{t}\| \boldsymbol{M} \cdot\left(\hat{\boldsymbol{t}} \times \frac{d \hat{\boldsymbol{t}}}{d S}\right) \quad m_{t}
\end{aligned}
$$

The components of the equilibrium Eq. (65) on the updated lo cal triad current $\{\hat{\boldsymbol{t}}, \hat{\boldsymbol{n}}, \hat{\boldsymbol{v}}\}$ are not relevant for the present work. The BC's of the equilibrium Eq. (65) projected on the local updated triad are

$$
\begin{aligned}
& \|\boldsymbol{t}\|\left(N+\frac{M_{n}}{R_{n}}+\frac{M_{v}}{R_{v}}\right)\left(L_{0}\right) \quad\|\boldsymbol{t}\| N_{r}\left(L_{0}\right) \quad F_{t}\left(L_{0}\right) \\
& \|\boldsymbol{t}\|\left(N+\frac{M_{n}}{R_{n}}+\frac{M_{v}}{R_{v}}\right)(0) \quad\|\boldsymbol{t}\| N_{r}(0) \quad F_{t}(0) \\
& \left(\frac{1}{\|\boldsymbol{t}\|} \frac{d}{d S}\left(\|\boldsymbol{t}\| M_{n}\right) \quad M_{v} \frac{\|\boldsymbol{t}\|}{\tau}+\frac{M_{t}}{R_{v}}\right)\left(L_{0}\right) \quad T_{v}\left(L_{0}\right) \quad F_{v}\left(L_{0}\right) \\
& \left(\frac{1}{\|\boldsymbol{t}\|} \frac{d}{d S}\left(\|\boldsymbol{t}\| M_{n}\right) \quad M_{v} \frac{\|\boldsymbol{t}\|}{\tau}+\frac{M_{t}}{R_{v}}\right)(0) \quad T_{v}(0) \quad F_{v}(0) \\
& \left(\frac{1}{\|\boldsymbol{t}\|} \frac{d}{d S}\left(\|\boldsymbol{t}\| M_{v}\right)+M_{n} \frac{\|\boldsymbol{t}\|}{\tau} \quad \frac{M_{t}}{R_{n}}\right)\left(L_{0}\right) \quad T_{n}\left(L_{0}\right) \quad F_{n}\left(L_{0}\right) \\
& \left(\frac{1}{\|\boldsymbol{t}\|} \frac{d}{d S}\left(\|\boldsymbol{t}\| M_{v}\right)+M_{n} \frac{\|\boldsymbol{t}\|}{\tau} \quad \frac{M_{t}}{R_{n}}\right)(0) \quad T_{n}(0) \quad F_{n}(0) \\
& \|\boldsymbol{t}\| M_{n}\left(L_{0}\right) \quad m_{n}\left(L_{0}\right) \\
& \|\boldsymbol{t}\| M_{n}(0) \quad m_{n}(0) \\
& \|\boldsymbol{t}\| M_{v}\left(L_{0}\right) \quad m_{v}\left(L_{0}\right) \\
& \|\boldsymbol{t}\| M_{v}(0) \quad m_{v}(0) \\
& M_{t}\left(L_{0}\right) \quad m_{t}\left(L_{0}\right) \\
& M_{t}(0) \quad m_{t}(0) .
\end{aligned}
$$

In the Eq. (66) have been introduced the definitions of the effec tive axial force $N_{r}$ and effective shear resultants $T_{n}$ and $T_{v}$.

\subsection{Constitutive operator of the rod}

We assume that the rod remains elastic. The stresses $\boldsymbol{S}$ can be obtained from an elastic potential, $\boldsymbol{S}=\nabla_{\boldsymbol{E}} \Psi(\boldsymbol{E})$, that, for isotropic materials, depends on the invariants $I_{E}, I I_{E}, I I I_{E}$ of the strain tensor. In this work we employ the approximation that the tangent elastic coefficients be constant, and according to Simo [22], Kiendl et al. [19], we use a quadratic form for the elastic potential,

$\Psi\left(I_{E}, I I_{E}, I I I_{E}\right) \quad \frac{1}{2}\left(a I_{E}^{2} \quad b I I_{E}\right)$.

Accounting for Kirchhoff Love constraints, it is $I_{E} \quad E_{t t}, I I_{E} \quad E_{t n}^{2} \quad E_{t v}^{2}$ so that

$\Psi(\boldsymbol{E}) \quad \frac{1}{2}\left(\mathrm{E} E_{t t}^{2}+2 \mathrm{G}\left(E_{t n}^{2}+E_{t v}^{2}\right)\right)$.

where the elastic moduli $\mathrm{E}$ and $\mathrm{G}$ have been introduced. It follows therefore

$$
S^{t t} \quad \mathrm{E} E_{t t} \quad S^{t n} \quad 2 \mathrm{G} E_{t n} \quad S^{t v} \quad 2 \mathrm{G} E_{t v}
$$

The stress resultants defined in (64) are calculated by means of the expressions

$$
\begin{aligned}
& N \quad \int_{\mathcal{A}} S^{t t} d \mathcal{A} \quad \frac{\mathrm{E} \mathcal{A}}{2}\left(\boldsymbol{t} \cdot \boldsymbol{t} \quad \boldsymbol{t}_{0} \cdot \boldsymbol{t}_{0}\right) \\
& M_{n} \quad \int_{\mathcal{A}} \vartheta^{v} S^{t t} d \mathcal{A} \quad \mathrm{E} \mathcal{I}_{n}\left(\begin{array}{ll}
\frac{\|\boldsymbol{t}\|^{2}}{R_{n}} & \frac{1}{R_{n 0}}
\end{array}\right) \\
& M_{v} \quad \int_{\mathcal{A}} \vartheta^{n} S^{t t} d \mathcal{A} \quad \mathrm{E} \mathcal{I}_{v}\left(\begin{array}{ll}
\frac{\|\boldsymbol{t}\|^{2}}{R_{v}} & \frac{1}{R_{v 0}}
\end{array}\right)
\end{aligned}
$$

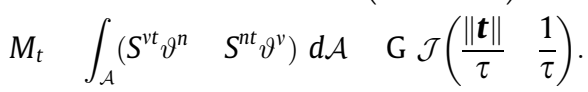

where the deformations are evaluated from the geometry of the up dated configuration. The equilibrium Eq. (65) are then used for eval uating the residual of equilibrium in an iterative procedure.

\section{Linearization}

The equilibrium Eq. (65) are non linear in $(\boldsymbol{u}, \phi)$, therefore an iterative procedure has to be used linearizing the equilibrium equation in the increments of the displacements and rotation.

Since the objective of this work is the application of B spline approximation to space rods, we consider only the case of small deformations, linearizing the equilibrium equations in $(\boldsymbol{u}, \phi)$. In this way, a linear system is obtained, that coincides with the material stiffness matrix referred to the undeformed configuration. It will be obtained from the principle of virtual power (63), using for the stresses the tangent constitutive equations:

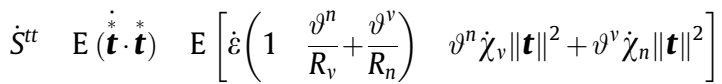

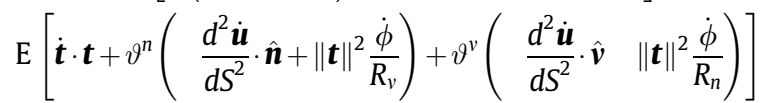

$$
\begin{aligned}
& \dot{S}^{t n} \quad G \vartheta^{v} \dot{\chi}_{t}\|\boldsymbol{t}\| \\
& \dot{S}^{t v} \quad G \vartheta^{n} \dot{\chi}_{t}\|\boldsymbol{t}\| \text {. }
\end{aligned}
$$

Substituting into the definitions of the sectional stress resul tants, and assuming, for simplicity, that the normal axes $\hat{\boldsymbol{n}}$ and $\hat{\boldsymbol{v}}$ be principal of inertia, one gets the following constitutive relations: 


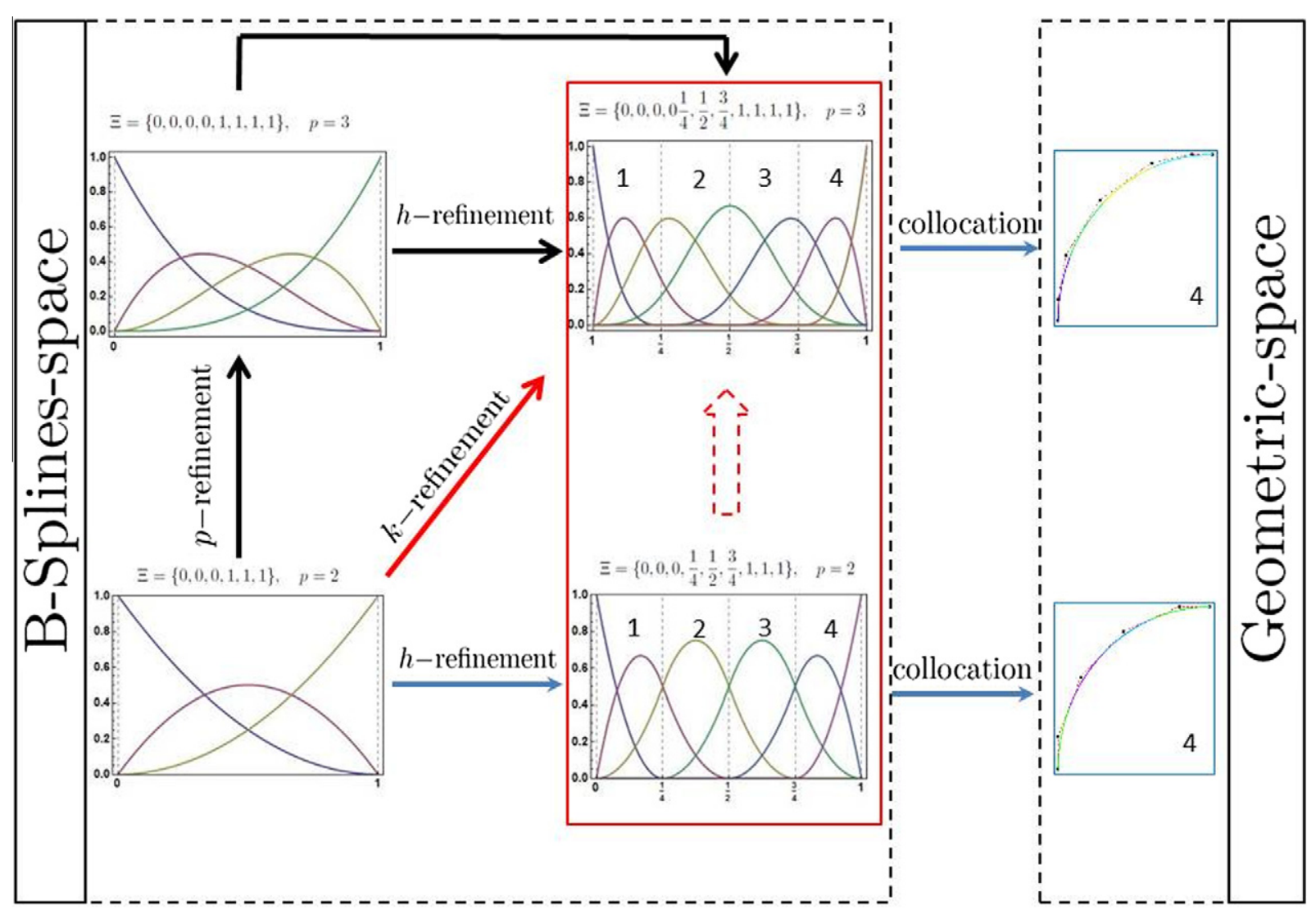

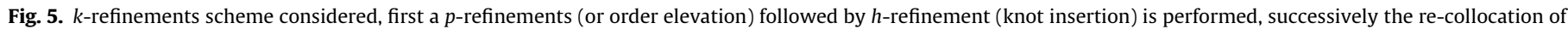
the control variable, corresponding to the new set of Greville abscissae, is performed.

$\left(\begin{array}{c}N_{r} \\ M_{n} \\ M_{v}\end{array}\right) \quad \mathrm{E}\left(\begin{array}{ccc}\mathcal{A}+\frac{\mathcal{I}_{n}}{R_{n}^{2}}+\frac{\mathcal{I}_{v}}{R_{v}^{2}} & \frac{\mathcal{I}_{n}}{R_{n}} & \frac{\mathcal{I}_{v}}{R_{v}} \\ \frac{\mathcal{I}_{n}}{R_{n}} & \mathcal{I}_{n} & 0 \\ \frac{\mathcal{I}_{v}}{R_{v}} & 0 & \mathcal{I}_{v}\end{array}\right)\left(\begin{array}{c}\dot{\varepsilon} \\ \dot{\chi}_{n}\|\boldsymbol{t}\|^{2} \\ \dot{\chi}_{v}\|\boldsymbol{t}\|^{2}\end{array}\right)$,

$M_{t} \quad G \quad \int_{\mathcal{A}}\left[\left(\vartheta^{n}\right)^{2}+\left(\vartheta^{v}\right)^{2}\right] d \mathcal{A} \dot{\gamma} \quad G \mathcal{J} \dot{\gamma}$

with $\mathcal{I}_{n} \quad \int_{\mathcal{A}}\left(\vartheta^{v}\right)^{2} d \mathcal{A}$ and $\mathcal{I}_{v} \quad \int_{\mathcal{A}}\left(\vartheta^{n}\right)^{2} d \mathcal{A}$.

In the case of infinitesimal deformation, $\|\boldsymbol{t}\| \rightarrow\left\|\boldsymbol{t}_{0}\right\|$ and $R_{n}, R_{v}$ become the curvatures of the reference configuration.

\section{Numerical formulation}

In the equilibrium equations derived in the previous sections the displacement functions are strongly coupled, involving differ ent degrees of differentiation. In order to guarantee continuity along the beam both the displacement functions and the geometric parameters of the axis curve, it is necessary that the interpolation be of a sufficiently high degree. In the paper we propose the use of a B spline bases interpolation, that have several advantages with respect to the traditional polynomial interpolation over finite elements.

\subsection{B Spline interpolation}

A B Spline curve of degree $p$ is defined as

$\mathbf{C}(\lambda) \quad \sum_{i=1}^{n} N_{i, p}(\lambda) \mathbf{P}_{i}$

where $\mathbf{P}_{i}=\left\{P_{i x}, P_{i y}, P_{i z}\right\}$ are the cartesian components of $n$ control points, and $N_{i, p}$ are the $n$ B Spline basis functions of degree $p$ defined on a non periodic knot vector. The knot vector is a non decreasing sequence of $m$ real numbers, the parametric coordinate $\lambda_{j}$, $j=1, \ldots, m$, with $m=n+p+1$,

$\Xi\{\underbrace{a, \ldots, a}_{p+1}, \underbrace{\lambda_{p+2}, \ldots, \lambda_{m(p+2)}}_{m 2(p+1)}, \underbrace{b, \ldots, b}_{p+1}\}$

The global interval $[a, b]$ is called the patch. The B Spline is said uniform if the knot vector are equally spaced, and non uniform otherwise. A knot vector is said open if the first and last knots have multiplicity $p+1$; in this work only non periodic open knot vectors are considered, with multiplicity equal to 1 for each internal knot, so that we have $C^{p}{ }^{1}$ parametric continuity at each knot. ${ }^{1}$

The basis functions $N_{i, p}(\lambda)$ are computed by means of the Cox deBoor recursion formula (see Piegl and Tiller [21]).

Starting from $p=0$ with

$N_{i, 0}(\lambda) \quad \begin{cases}1, & \text { if } \lambda \in\left[\lambda_{i}, \lambda_{i+1}[\right. \\ 0, & \text { otherwise. }\end{cases}$

for $p \geqslant 1$ we have:

$N_{i, p}(\lambda) \begin{cases}\frac{\lambda \lambda_{i}}{\lambda_{i+p} \lambda_{i}} N_{i, p} 1(\lambda)+\frac{\lambda_{i+p+1} \lambda}{\lambda_{i+p+1} \lambda_{i+1}} N_{i+1, p} 1(\lambda), & \text { if } \lambda \in\left[\lambda_{i}, \lambda_{i+p+1}[,\right. \\ 0, & \text { otherwise. }\end{cases}$

In the formula (76) the position $0 / 0=0$ must be considered. The generic interval $\left[\lambda_{i}, \lambda_{i+1}[\right.$ is called the knot span and can have zero length; knot spans with length different from zero are called sec tions. For an open knot vector there are $m 2(p+1)+1$ sections.

\footnotetext{
${ }^{1}$ If a knot has multiplicity $k$ the B-Spline has $C^{p k}$ continuity at the knot, i.e. if the multiplicity is $k=p$ the $\mathrm{B}-$ Spline has $C^{0}$ continuity at the knot considered.
} 


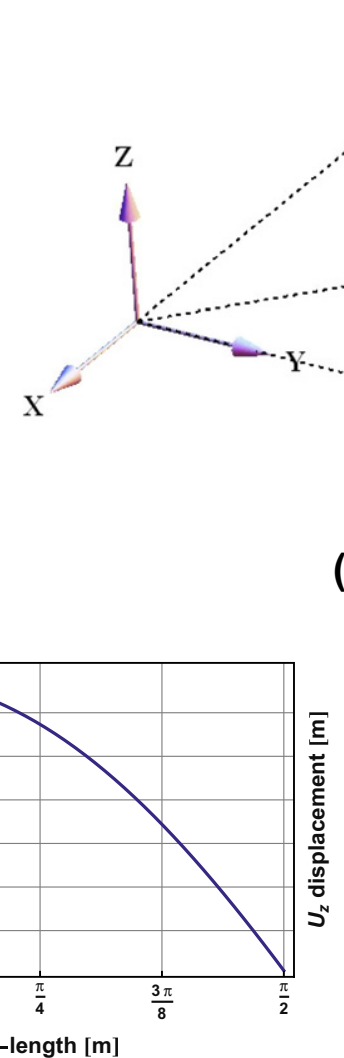

(b)

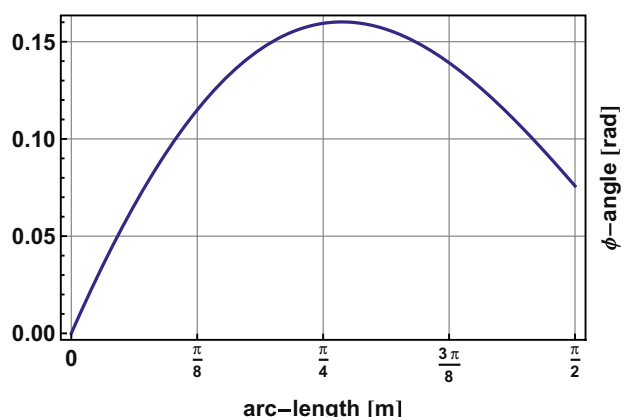

(c)

Fig. 6. Clamped torsion-free space arch; (a) initial geometry of the rod; (b) solution for the $u_{z}(S)$ displacement and (c) solution for the correction-angle $\phi(S)$.

In each section there are $p+1$ splines different from zero. Fig. 3 shows an example of $B$ splines bases on the interval $[0,1]$.

If in the knot vector there are not internal knots the basis func tions reduce to the Bernstein polynomials, so that the B Spline interpolation is a generalization of the Bezier's interpolation. An interesting property of the B Spline interpolation of a curve with an open knot vector is that the curve is tangent to the control poly gon at the ends.

\subsection{Representation of the geometry: collocation and refinements}

In this work we use an isoparametric B Spline representation, that is, both the reference geometry and the configuration vari ables are approximated by means of the same B spline bases. In this manner one of the interesting property of isogeometric analy sis, the internal high continuity, is preserved. We do not follow the usual isogeometric approach, that employs NURBS for the approx imation of the relevant variables.

A NURBS curve is the projection of the four dimensional B Spline of $\mathbb{R}^{4}$ onto $\mathbb{R}^{3}$, in this sense all the property for B Spline are transferred to NURBS. In this approach the initial geometry is described exactly, and it is invariant under refinements. The basis functions are weighted and non rational and during the mechanical simulation the weights remain fixed.

In our approach, in order to reduce the error, $h$ or $k$ refine ments are used on the initial geometry as well as on the configura tion parameters space, analogously to the refinement strategy of the standard FEM codes.

Hughes et al. [14] and Cottrell et al. [9] have examined some of the most common refinement techniques in isogeometric analysis, the fundamental being the $h$ refinement, the $p$ refinement and the $k$ refinement. Under $h$ refinement, or knot insertion, if the multi plicity of the inserted knot is one, the geometry and the internal continuity are invariants; while under $k$ refinement, or degree ele vation in the sense of the polynomial and continuity degree, only the geometry is invariant, but the continuity is increased consis tently to the polynomial degree.

In this work we use $h$ refinement and $k$ refinement. The $h$ refinement is described in Fig. 4 . A standard knot insertion is per formed in the B Splines space, see Piegl and Tiller [21]. Only knots with multiplicity one are inserted, so that the continuity degree remains unchanged. The newly generated control variables are then collocated in the Greville abscissas and the geometry is newly de fined. Alternative sets of collocation abscissae are presented and analyzed in [3], for the collocation of strong differential operators.

In the $k$ refinement, a $p$ refinement (polynomial order eleva tion) is performed in the B Splines spaces, followed by an $h$ refine ment (knot insertion), see Hughes et al. [14] and Cottrell et al. [9]. In this manner one gets higher continuity and polynomial order, see Fig. 5.

\subsection{B Spline interpolation of the rod}

The interpolation of a configuration of the rod is obtained as functions of the vector $\mathbf{q}$ that collects the coordinates of the control points and the twist angle:

$\mathbf{q}(\lambda) \quad\left\{p_{x}(\lambda), p_{y}(\lambda), p_{z}(\lambda), \phi(\lambda)\right\}$

$p_{\alpha}(\lambda) \quad \sum_{i=1}^{n} N_{i, p} P_{\alpha i} \quad \hat{\mathbb{M}} \boldsymbol{P}_{\alpha} \phi(\lambda) \quad \sum_{i=1}^{n} N_{i, p} \Phi_{i} \quad \hat{\mathbf{M}} \boldsymbol{\Phi}$ 


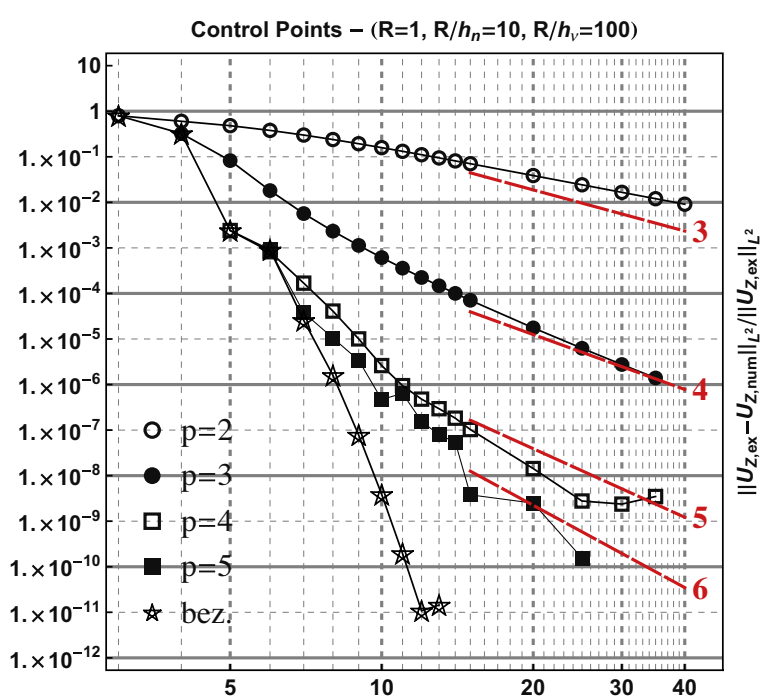

(a)

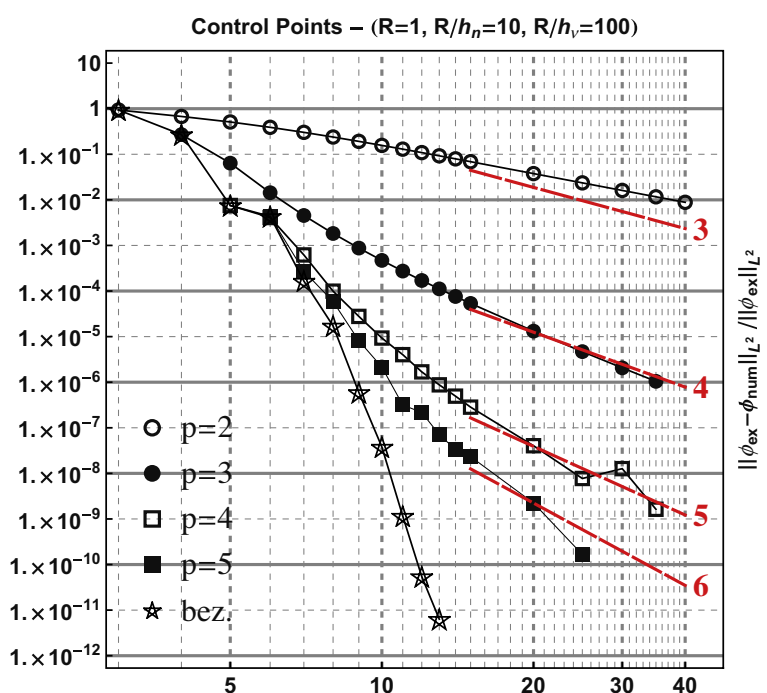

(b)

Fig. 7. Cantilever space arch; (a) relative error in $L^{2}$-norm for the displacement's component $u_{z}$ and (b) relative error in $L^{2}$-norm for the correction angle $\phi$ for different polynomial degree.

so that $\boldsymbol{p}(\lambda) \quad M P$ with

$\mathbb{M}\left[\begin{array}{ccc}\hat{M} & 0 & 0 \\ 0 & \hat{M} & 0 \\ 0 & 0 & \hat{M}\end{array}\right]$

where the matrix $\hat{\mathbb{M}}\left[N_{1, p}, \ldots, N_{n, p}\right]$. In general it is more conve nient to perform the interpolation sectiontwise, since on each seg ment only $p+1$ basis splines are different from zero. However, in order to simplify the exposition, we refer to the interpolation over the whole patch, as happens in the case of Bezier interpolation. We define the following matrices of the derivatives of the interpo lation functions

$\mathbb{B} \frac{d \mathbb{M}}{d \lambda} \mathbb{D} \frac{d^{2} \mathbb{M}}{d \lambda^{2}}$

The interpolation of the initial tangent vector and of its norm is

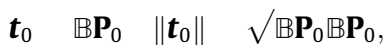

that are evaluated on the interpolation of the initial geometry. The interpolated arc length differentiation is

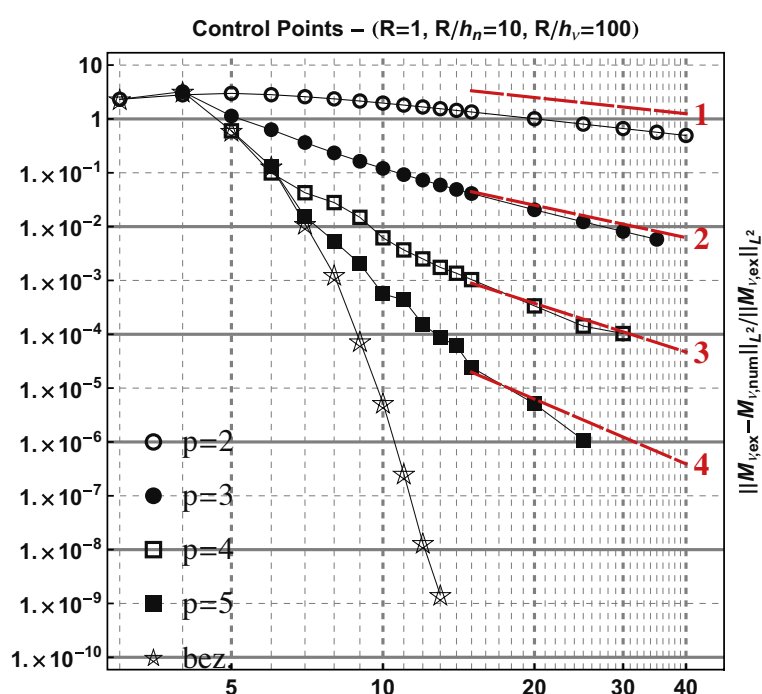

(a)

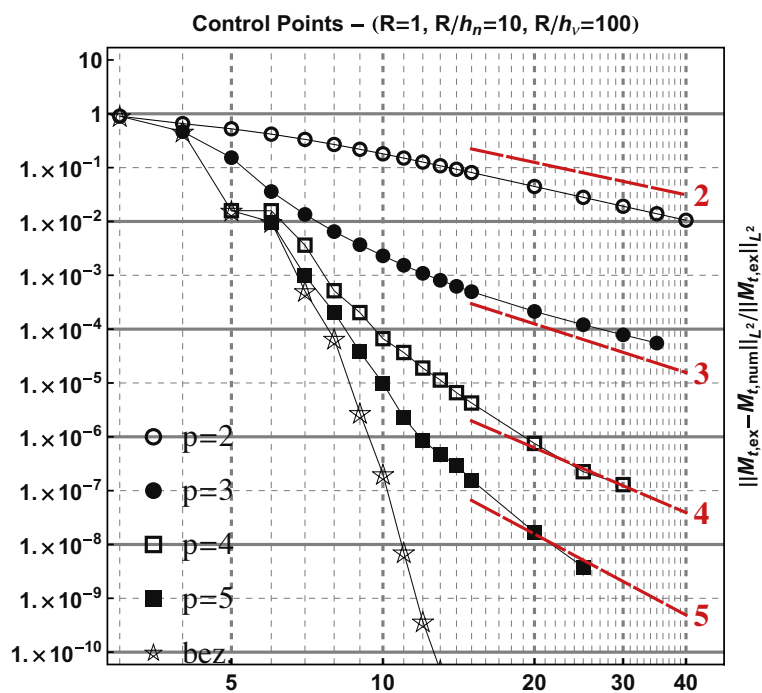

(b)

Fig. 8. Cantilever space arch; (a) relative error in $L^{2}$-norm for the bending moment $M_{v}$ and (b) relative error in $L^{2}$-norm for the twisting moment $M_{t}$ for different polynomial degree.

$\frac{d(\bullet)}{d S} \frac{\mathbb{B}}{\sqrt{ } \mathbf{P}^{T} \mathbb{B}^{T} \mathbb{B} \mathbf{P}}$.

so that the cartesian components of the current tangent vector is gi ven by

$$
\text { t } \frac{\mathbb{B P}}{\left\|\boldsymbol{t}_{0}\right\|}
$$

The interpolation of the second gradient along the arc length, according to expression (23) is

$$
\begin{aligned}
\frac{d^{2} \bullet}{d S^{2}} \quad \frac{1}{\left\|\boldsymbol{t}_{0}\right\|} \frac{d}{d \lambda}\left(\frac{1}{\left\|\boldsymbol{t}_{0}\right\|} \frac{d \bullet}{d \lambda}\right) \quad \frac{1}{\left\|\boldsymbol{t}_{0}\right\|^{2}} \frac{1}{\|\boldsymbol{t}\|^{2}}\left(\frac{d \boldsymbol{t}}{d \lambda} \cdot \boldsymbol{t}\right) \frac{d \bullet}{d \lambda} \\
\times \frac{1}{\left\|\boldsymbol{t}_{0}\right\|^{2}} \mathbb{D} \quad \frac{\left(\mathbb{D} \mathbf{P}_{0} \cdot \mathbb{B} \mathbf{P}_{0}\right)}{\left\|\boldsymbol{t}_{0}\right\|^{4}} \mathbb{B} \quad \frac{1}{\mathbb{B} \mathbf{P} \cdot \mathbb{B} \mathbf{P}} \frac{1}{\left\|\boldsymbol{t}_{0}\right\|^{2}} \\
\times\left[\mathbb{D P} \cdot \mathbb{B} \mathbf{P} \quad \mathbb{B} \mathbf{P} \cdot \mathbb{B} \mathbf{P} \frac{\mathbb{D} \mathbf{P}_{0} \cdot \mathbb{B} \mathbf{P}_{0}}{\mathbb{B} \mathbf{P}_{0} \cdot \mathbb{B} \mathbf{P}_{0}}\right] \mathbb{B} \\
\quad \times \frac{1}{\left\|\boldsymbol{t}_{0}\right\|^{2}} \mathbb{D} \quad \frac{\mathbb{D} \mathbf{P} \cdot \mathbb{B} \mathbf{P}}{\|\boldsymbol{t}\|^{2}\left\|\boldsymbol{t}_{0}\right\|^{2}} \mathbb{B} \quad \mathbb{X}
\end{aligned}
$$




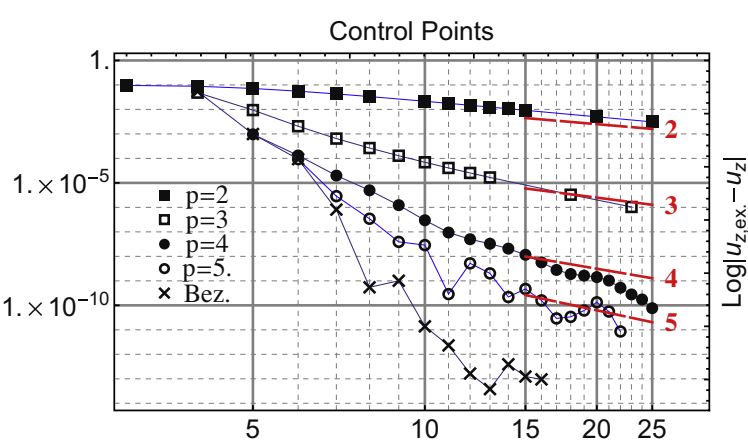

(a)

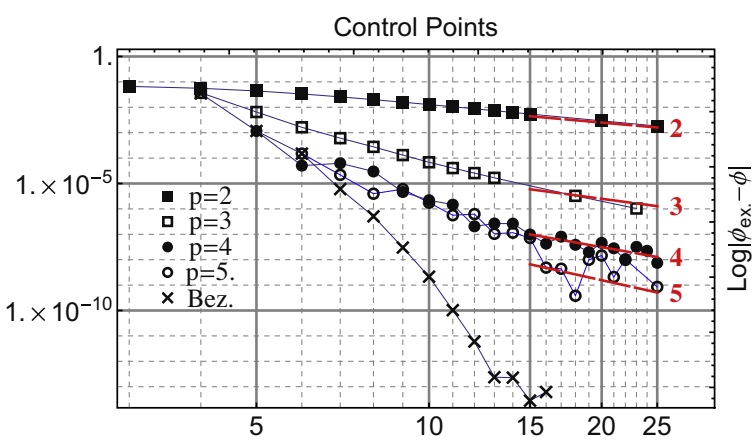

(b)

Fig. 9. (a) Error on the displacement $\boldsymbol{u}_{z}(L)$; (b) error on the torsion angle $\phi(L)$.

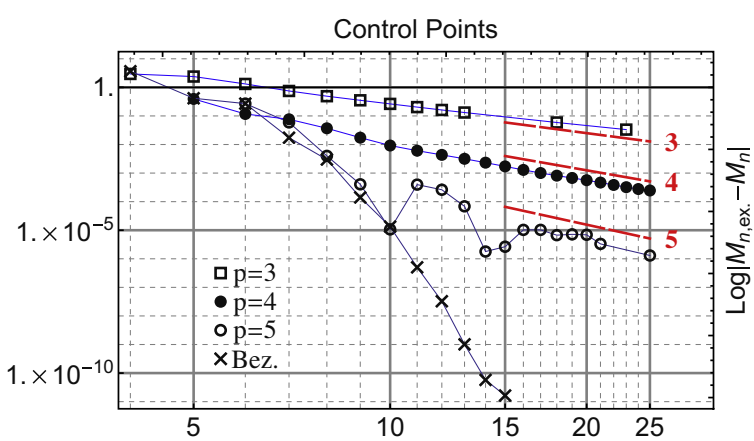

(a)

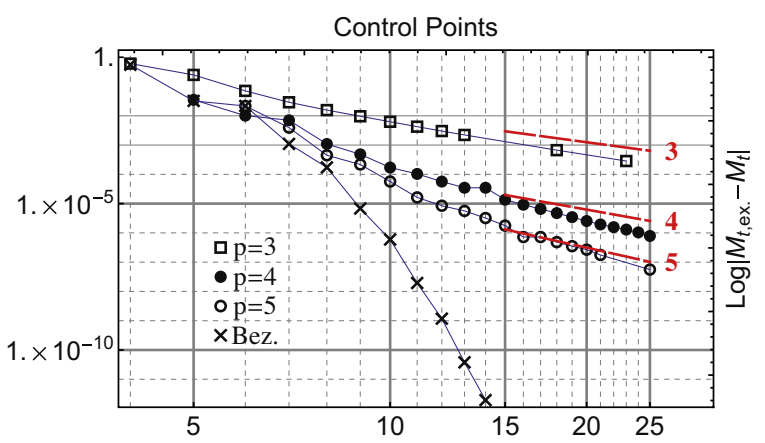

(b)

Fig. 10. (a) Error on the bending moment at the first end; (b) error on the twisting moment at the first end.
The cartesian components of the sectional axes $\hat{\boldsymbol{n}}, \hat{\boldsymbol{v}}$ are given by expressions (13) once the interpolation of the tangent vector (82) has been obtained, and will be indicated as

$\begin{array}{llll}\boldsymbol{n} & \mathbb{N}(\mathbf{P}) & \boldsymbol{v} & \mathbb{V}(\mathbf{P}) .\end{array}$

\subsection{Material stiffness matrix}

The material (tangent) stiffness matrix of the rod is derived from the virtual power expression (63) using the definitions (64) of the stress resultants and the constitutive Eq. (71) for the stress components. We shall examine each contribution to the internal power separately.

\subsubsection{Material axial stiffness}

The axial stiffness is obtained from the following integral, where a tilde denotes virtual deformations:

$\int_{L_{0}} \mathrm{E} \mathcal{A} \dot{\varepsilon} \tilde{\varepsilon} d S \quad \int_{0}^{L_{0}} \mathrm{E} \mathcal{A}\left(\boldsymbol{t} \cdot \frac{d \dot{\boldsymbol{u}}}{d S}\right)\left(\boldsymbol{t} \cdot \frac{d \tilde{\boldsymbol{u}}}{d S}\right)\left\|\boldsymbol{t}_{0}\right\| d \lambda$

Using the approximation $\boldsymbol{t} \frac{\mathbb{B P}}{\left\|\boldsymbol{t}_{0}\right\|}$, the axial stiffness matrix gets the form

$K_{a x} \quad\left[\begin{array}{cc}\int_{0}^{L_{0}} \mathrm{E} \mathcal{A} \frac{\left(\mathbb{B}^{T} \mathbb{B} \mathbf{P}\right)^{T}\left(\mathbb{B}^{T} \mathbb{B P}\right)}{\left\|\boldsymbol{t}_{0}\right\|^{3}} d \lambda & \mathbf{0} \\ \mathbf{0} & \mathbf{0}\end{array}\right]\left[\begin{array}{c}\Delta \boldsymbol{P} \\ \Delta \boldsymbol{\Phi}\end{array}\right]$

\subsubsection{Material bending stiffness}

Since the axes $\hat{\boldsymbol{n}}, \hat{\boldsymbol{v}}$ have been chosen principal of inertia, the bending material stiffness is obtained from the expression

$$
\begin{aligned}
& \int_{0}^{L_{0}} \mathrm{E} \mathcal{I}_{v}\left(\frac{d^{2} \dot{\boldsymbol{u}}}{d S^{2}} \cdot \hat{\boldsymbol{n}}+\hat{\boldsymbol{v}} \cdot \frac{d \hat{\boldsymbol{t}}}{d S}\|\boldsymbol{t}\| \dot{\phi}\right)\left(\frac{d^{2} \tilde{\boldsymbol{u}}}{d S^{2}} \cdot \hat{\boldsymbol{n}}+\hat{\boldsymbol{v}} \cdot \frac{d \hat{\boldsymbol{t}}}{d S}\|\boldsymbol{t}\| \tilde{\phi}\right) \\
& +\int_{0}^{L_{0}} \mathrm{E} \mathcal{I}_{n}\left(\frac{d^{2} \dot{\boldsymbol{u}}}{d S^{2}} \cdot \hat{\boldsymbol{v}}+\hat{\boldsymbol{n}} \cdot \frac{d \hat{\boldsymbol{t}}}{d S}\|\boldsymbol{t}\| \dot{\phi}\right)\left(\frac{d^{2} \tilde{\boldsymbol{u}}}{d S^{2}} \cdot \hat{\boldsymbol{v}}+\hat{\boldsymbol{n}} \cdot \frac{d \hat{\boldsymbol{t}}}{d S}\|\boldsymbol{t}\| \tilde{\phi}\right)
\end{aligned}
$$

The geometric curvatures are interpolated as follows

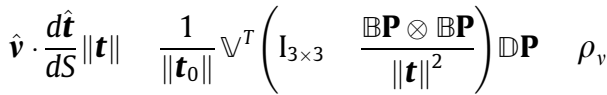

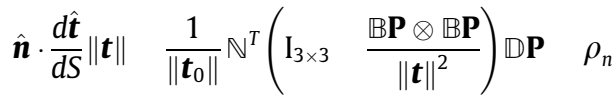

The bending velocity of curvature operators are given by

$$
\begin{aligned}
& \frac{d^{2} \dot{\boldsymbol{u}}}{d S^{2}} \cdot \hat{\boldsymbol{n}}+\hat{\boldsymbol{v}} \cdot \frac{d \hat{\boldsymbol{t}}}{d S}\|\boldsymbol{t}\| \dot{\phi} \quad \mathbb{N}^{T} \backslash \dot{\boldsymbol{d}}+\rho_{v} \hat{M} \dot{\boldsymbol{\Phi}} \\
& \frac{d^{2} \dot{\boldsymbol{u}}}{d S^{2}} \cdot \hat{\boldsymbol{v}}+\hat{\boldsymbol{n}} \cdot \frac{d \hat{\boldsymbol{t}}}{d S}\|\boldsymbol{t}\| \dot{\phi} \quad \mathbb{V}^{T} \backslash \dot{\boldsymbol{d}}+\rho_{n} \hat{\mathbb{M}} \dot{\boldsymbol{\Phi}}
\end{aligned}
$$

The material bending stiffness is therefore given by the sum of the two matrices:

$$
\begin{array}{ll}
K_{\hat{n}} & \mathrm{E} \mathcal{I}_{v} \int_{0}^{L_{0}}\left[\begin{array}{cc}
\mathbb{X}^{T} \mathbb{N}^{T} \mathbb{X} & \rho_{v} \mathbb{X}^{T} \mathbb{N} \hat{M} \\
\rho_{v} \hat{\mathbb{M}}^{T} \mathbb{N}^{T} \mathbb{X} & \rho_{v}^{2} \hat{\mathbb{M}}^{T} \hat{\mathbb{M}}
\end{array}\right]\left[\begin{array}{c}
\Delta \boldsymbol{P} \\
\Delta \boldsymbol{\Phi}
\end{array}\right] \\
K_{\hat{v}} & \mathrm{E} \mathcal{I}_{n} \int_{0}^{L_{0}}\left[\begin{array}{cc}
\mathbb{X}^{T} \mathbb{V} \mathbb{V}^{T} \mathbb{X} & \rho_{n} \mathbb{X}^{T} \mathbb{V} \hat{\mathbb{M}} \\
\rho_{n} \hat{\mathbb{M}}^{T} \mathbb{V}^{T} \mathbb{X} & \rho_{n}^{2} \hat{\mathbb{M}}^{T} \hat{\mathbb{M}}
\end{array}\right]\left[\begin{array}{c}
\Delta \boldsymbol{P} \\
\Delta \boldsymbol{\Phi}
\end{array}\right]
\end{array}
$$

\subsubsection{Material torsional stiffness}

The torsional internal power is

$\int_{0}^{L_{0}} \mathrm{G} \mathcal{J} \dot{\chi}_{t} \tilde{\chi}_{t}\left\|\boldsymbol{t}_{0}\right\| d \lambda$ 


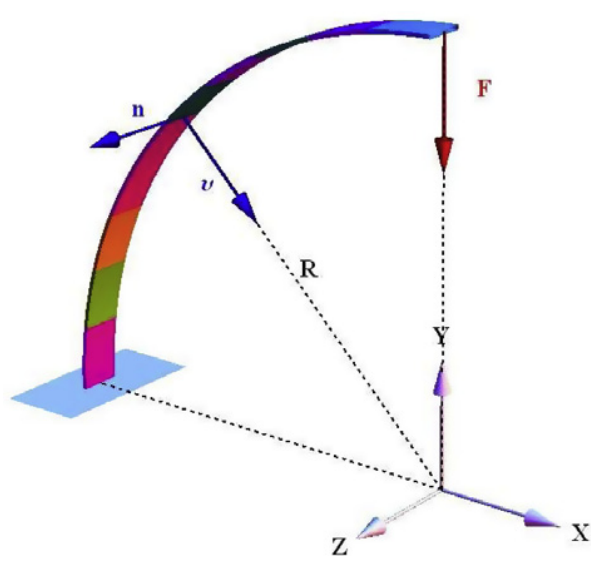

(a)

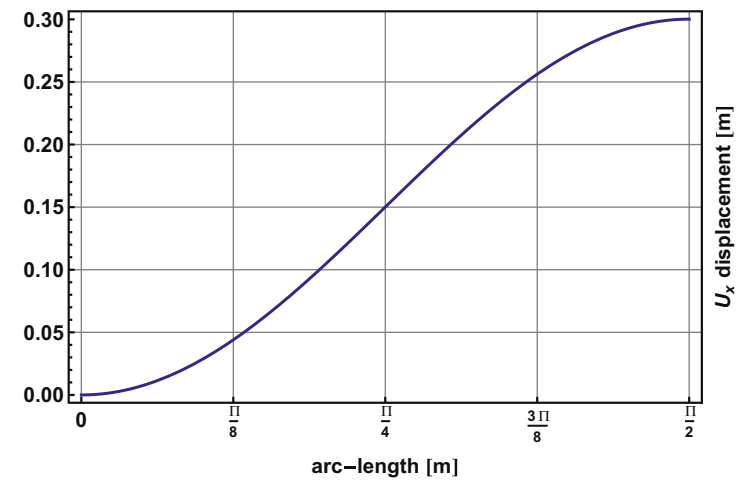

(b)

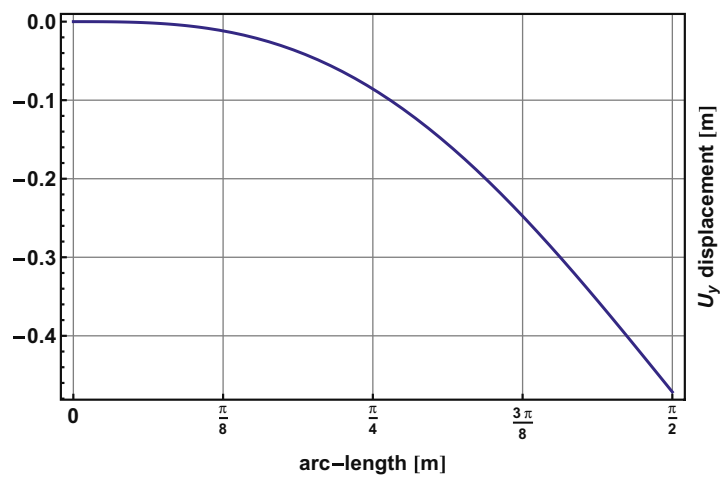

(c)

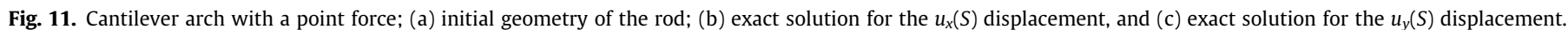

The interpolation of the torsional velocity of curvature is

$$
\begin{aligned}
& \frac{1}{\|\boldsymbol{t}\|}\left(\hat{\boldsymbol{t}} \times \frac{d \hat{\boldsymbol{t}}}{d S}\right) \cdot \frac{d \dot{\boldsymbol{u}}}{d S}+\frac{d \dot{\phi}}{d S} \quad \frac{1}{\|\boldsymbol{t}\|}\left(\hat{\boldsymbol{v}} \cdot \frac{d \hat{\boldsymbol{t}}}{d S} \frac{d \dot{\boldsymbol{u}}}{d S} \cdot \hat{\boldsymbol{n}}+\hat{\boldsymbol{n}} \cdot \frac{d \hat{\boldsymbol{t}}}{d S} \frac{d \dot{\boldsymbol{u}}}{d S} \cdot \hat{\boldsymbol{v}}\right) \\
& +\frac{d \dot{\phi}}{d S} \quad \frac{1}{\left\|\boldsymbol{t}_{0}\right\|} \frac{1}{\|\boldsymbol{t}\|^{2}}\left(\rho_{v} \mathbb{N}^{T}+\rho_{n} \mathbb{V}^{T}\right) \mathbb{B} \dot{\boldsymbol{P}} \\
& +\frac{1}{\left\|\boldsymbol{t}_{0}\right\|} \hat{\mathbb{B} \dot{\boldsymbol{\Phi}}} \quad \frac{1}{\left\|\boldsymbol{t}_{0}\right\|} \mathbb{X}_{t} \dot{\boldsymbol{P}}+\frac{1}{\left\|\boldsymbol{t}_{0}\right\|} \hat{\mathbb{B}} \dot{\boldsymbol{\Phi}} .
\end{aligned}
$$

The material torsional stiffness matrix is therefore

$$
K_{t} \quad G \mathcal{J} \int_{0}^{L_{0}} \frac{1}{\left\|\boldsymbol{t}_{0}\right\|^{2}}\left[\begin{array}{cc}
\mathbb{X}_{t}^{T} \mathbb{X}_{t} & \mathbb{X}_{t}^{T} \hat{\mathbb{B}} \\
\hat{\mathbb{B}}^{T} \mathbb{X}_{t} & \hat{\mathbb{B}}^{T} \hat{\mathbb{B}}
\end{array}\right]\left[\begin{array}{c}
\Delta \boldsymbol{P} \\
\Delta \boldsymbol{\Phi}
\end{array}\right]
$$

\subsection{Numerical integration}

Gauss Siedel and Gauss Lobatto quadrature rules have been used, considering $p+1$ Gauss quadrature points per section. No significative difference has been found between the two quadra ture rules.

\section{Numerical applications}

In this section some applications are presented, for checking the numerical model presented. Several geometries are tested, in order to prove that the performance of the model does not depend on the particular geometry studied. For the simpler academic examples, a convergence analysis is also presented.

\subsection{Cantilever space arch}

The first example concerns the horizontal arch shown in Fig. 6(a), clamped at the first end and subjected to a vertical force $\boldsymbol{F}=\{0,0,1\},[k N]$ at the free end. For this example it is possible to evaluate the exact solution, so a convergence analysis for the exact error will be presented. Both the convergence for the $L^{2}$ norm of the error and for some relevant values of the displacements or stresses will be examined.

The radius of the centroid curve is $R=1[\mathrm{~m}]$ the section is rect angular with $h_{n}=0.1$ and $h_{v}=0.01[\mathrm{~m}]$ respectively, and $\mathrm{E}=1.999 * 10^{8}\left[\mathrm{kN} / \mathrm{m}^{2}\right], v=0.25,(G=\mathrm{E} /(2+2 v))$. The centroid curve is given by

$$
\begin{array}{cccccc}
P_{x}(\alpha) \quad R \cos [\pi & \alpha
\end{array} \quad P_{y}(\alpha) \quad R \sin \left[\begin{array}{ll}
\pi & \alpha
\end{array}\right] \quad P_{z}(\alpha) \quad 0,
$$

No geometrical torsion is considered in this case, that is $\phi_{0}(S)=0 \forall S \in\left[0, L_{0}\right]$. The initial unit tangent vector at the origin is $\hat{\boldsymbol{t}}_{\mathbf{0}} \quad\{0,1,0\}$ and the unit normal vectors are assumed as $\hat{\boldsymbol{n}}_{\mathbf{0}}(0) \quad\{0,0,1\}$ and $\hat{\boldsymbol{v}}_{\mathbf{0}}(0) \quad \hat{\boldsymbol{t}}_{\mathbf{0}}(0) \times \hat{\boldsymbol{n}}_{\mathbf{0}}(0) \quad\{1,0,0\}$. Since in this case it is $\boldsymbol{R}\left(\phi_{0}, \hat{\boldsymbol{t}}_{\mathbf{0}}(S)\right) \quad \boldsymbol{I}$ everywhere it results $\hat{\boldsymbol{n}}_{\mathbf{0}}(S) \quad \hat{\boldsymbol{n}}_{\mathbf{0}}(0)$ and $\hat{\boldsymbol{v}}_{\mathbf{0}}(S) \quad \boldsymbol{\Lambda}_{0}\left(\hat{\boldsymbol{t}}_{0}(0), \hat{\boldsymbol{t}}_{0}(S)\right) \hat{\boldsymbol{n}}_{\mathbf{0}}(0)$. The boundary conditions at the first end are given by 


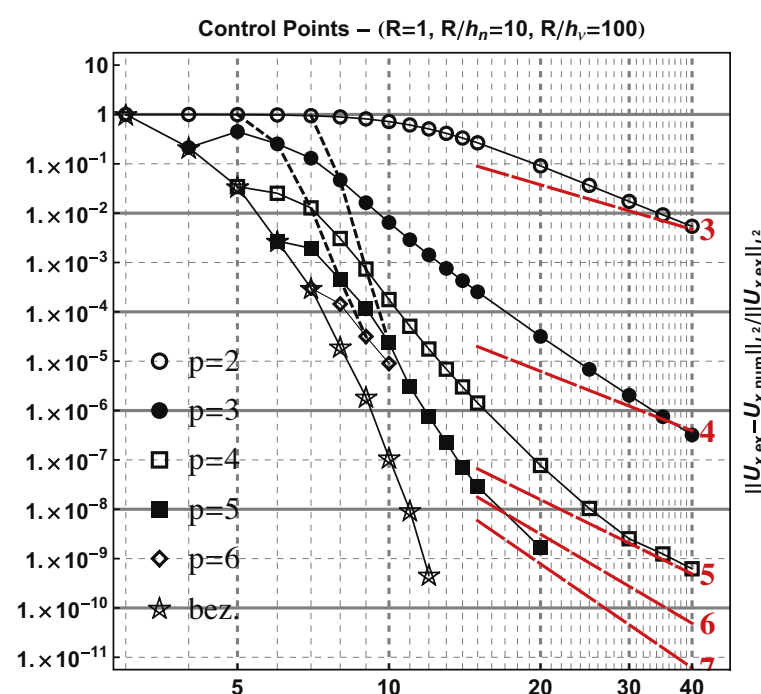

(a)

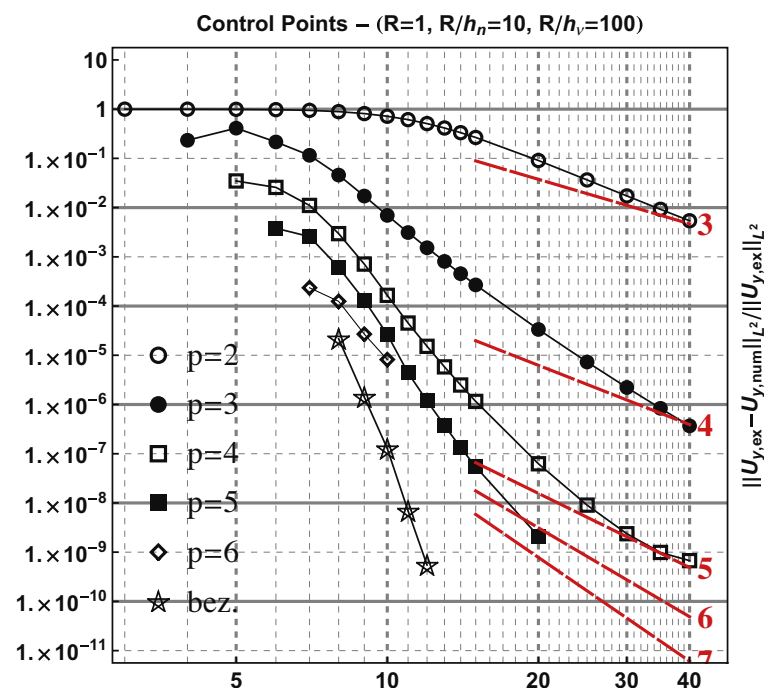

(b)

Fig. 12. 2D-cantilever arch with a point force at the end; (a) relative error in $L^{2}$ norm for the displacement's component $u_{x}$ and (b) relative error in $L^{2}$-norm for the displacement's component $u_{y}$ for different polynomial degrees.

$\dot{\boldsymbol{u}}(0$

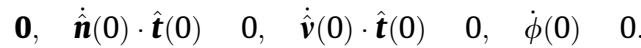

Setting $\mathcal{I}_{v} \frac{h_{v} h_{n}^{3}}{12}, \mathcal{I}_{n} \quad \frac{h_{v}^{3} h_{n}}{12}$ and $\mathcal{J} \quad \frac{h_{v}^{3} h_{n}}{3}$ the exact solution for the vertical displacement $u_{z}(S)$ and for the torsional rotation $\phi(S)$ are given respectively by

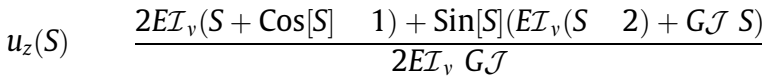

$$
\begin{aligned}
& \phi(S) \quad \frac{\operatorname{Sin}[S]\left(E \mathcal{I}_{v}(S \quad 2)+G \mathcal{J} S\right)}{2 E \mathcal{I}_{v} G \mathcal{J}}
\end{aligned}
$$

At the free end we find

$$
\begin{array}{lrrrr}
u_{z}(\pi / 2) & \frac{\pi}{4 E \mathcal{I}_{v}} & \frac{3 \pi}{4 G \mathcal{J}}+\frac{2}{G \mathcal{J}} & 0.13835450005254035 & {[\mathrm{~m}],} \\
\phi(\pi / 2) & \frac{\pi}{4 E \mathcal{I}_{v}} & \frac{\pi}{4 G \mathcal{J}}+\frac{1}{G \mathcal{J}} & 0.07580120034574506 & {[\mathrm{rad}] .}
\end{array}
$$

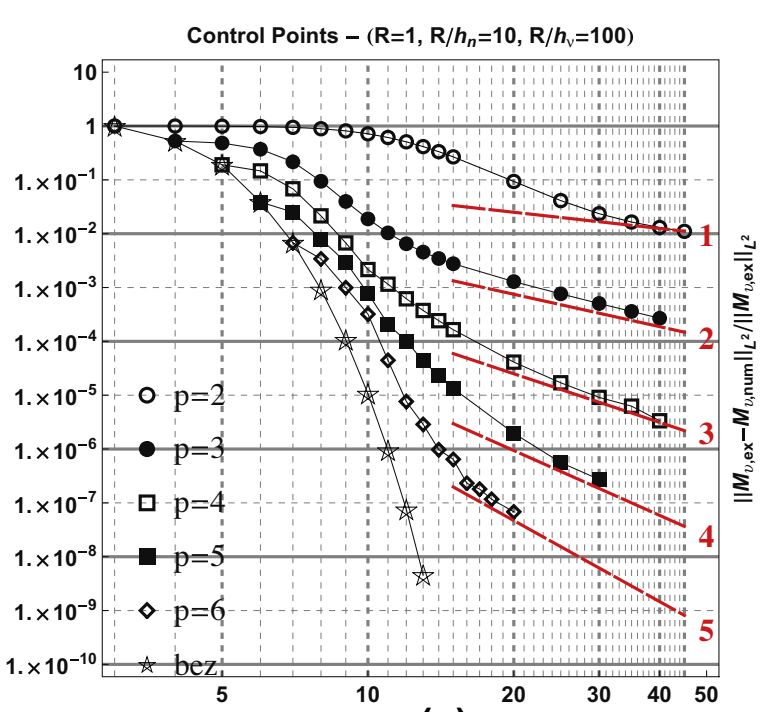

(a)

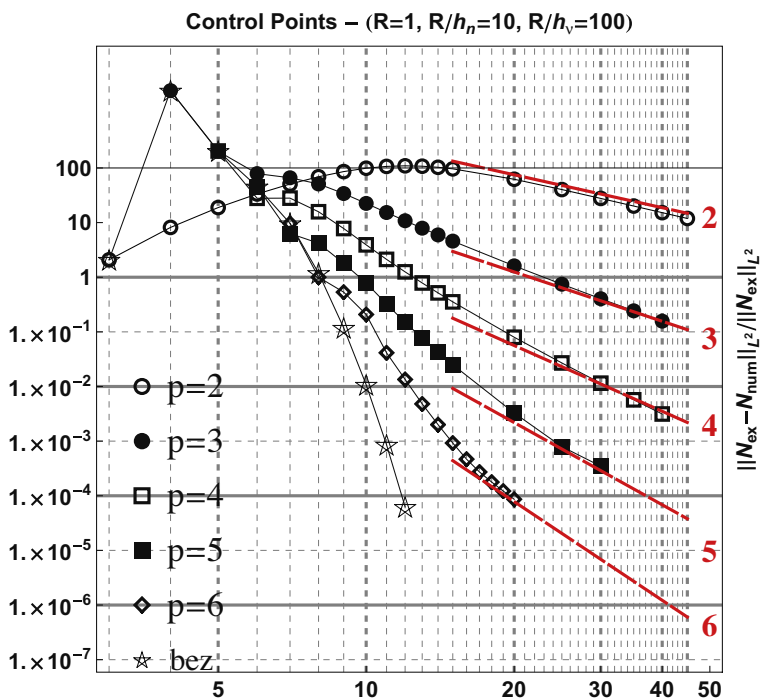

(b)

Fig. 13. 2D-cantilever arch with a point force at the end; (a) relative error in $\mathrm{L}^{2}$ norm for the bending moment $M_{v}$ and (b) relative error in $L^{2}$-norm for the axial force $N$, for different polynomial degrees.

The initial geometry and the deformation fields have been rep resented by B splines of degree ranging from 2 to 5 . For each poly nomial degree an increasing number of degrees of freedom has been considered, inserting internal knots by means of the $h$ refine ment procedure. Up to 40 control points have been considered. In addition, for comparison, the problem has been solved using Be zier's interpolation, with polynomial degree from 2 to 12 .

In Fig. 6(b) and (c) are plotted the vertical displacement $u_{z}(S)$ and the torsional rotation $\phi(S)$ numerically calculated using 5th degree B splines. The results obtained are very close to the exact solution.

Convergence plots for the vertical displacement, torsional rota tion, bending and torsional moment are reported in Figs. 7(a), (b), $8(a)$ and (b). On the horizontal axis is reported the number of con trol points used, proportional to the dimension of the approxima tion space. On the vertical axis is reported the $L^{2}$ error norm. 


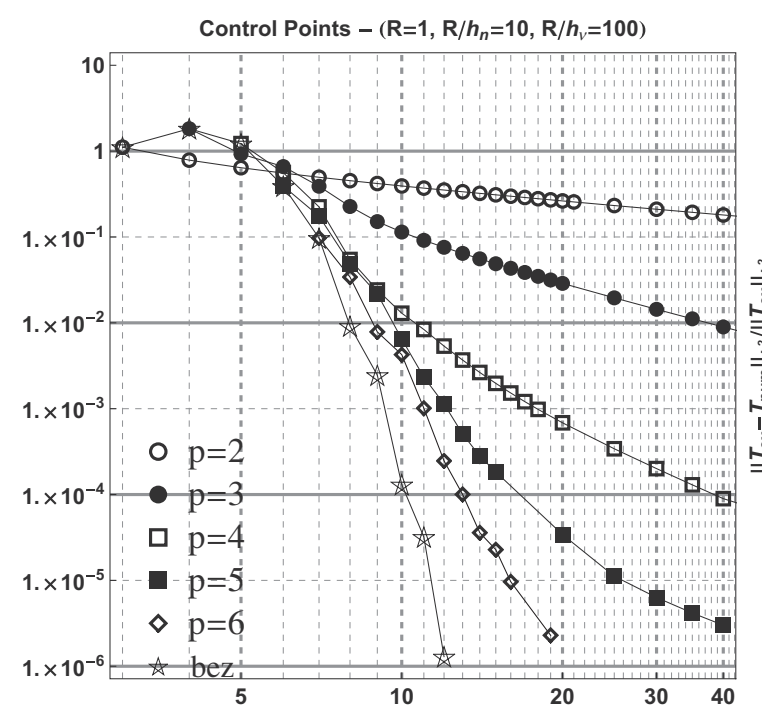

(a)

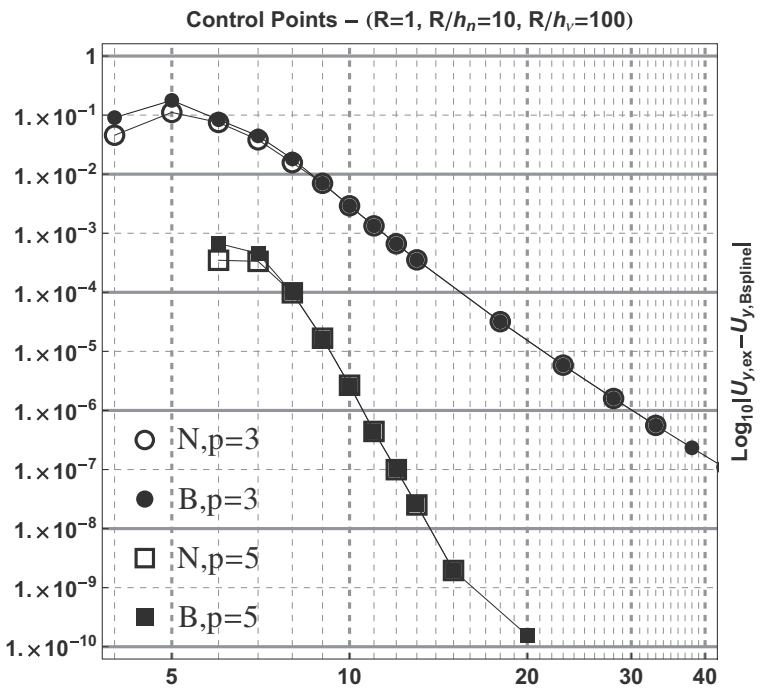

(b)

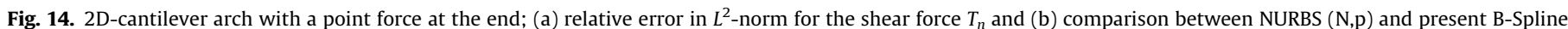
analysis (B,p), for two polynomial degrees.

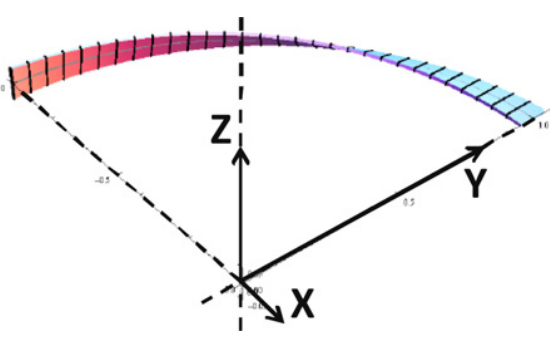

(a)

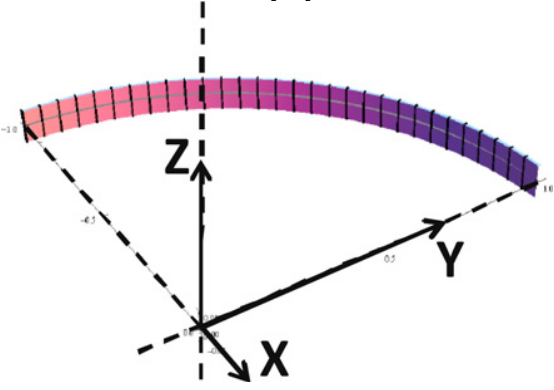

(c)

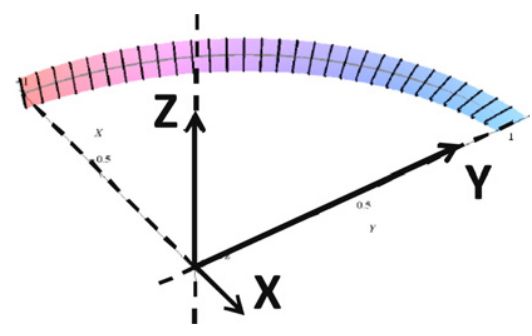

(e)

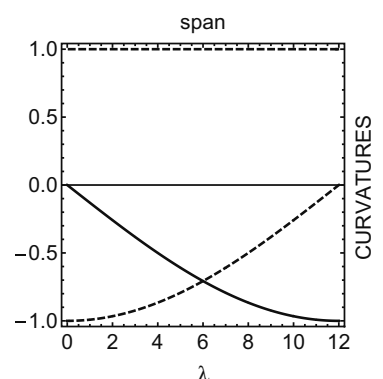

(b)

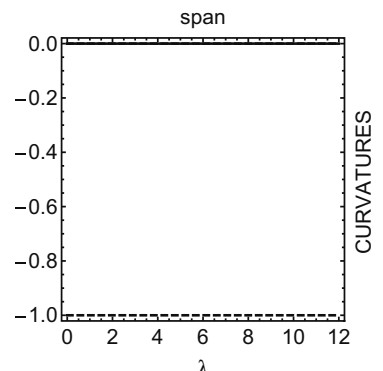

(d)

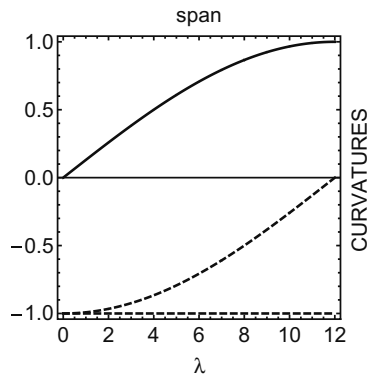

(f)

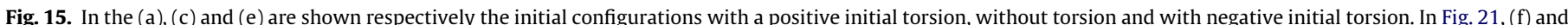

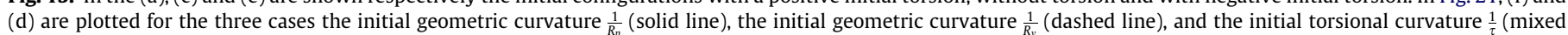
line). 


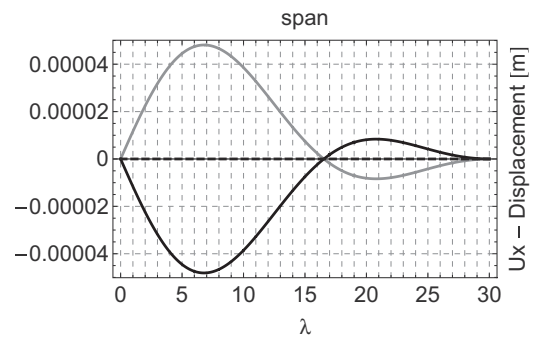

(a)

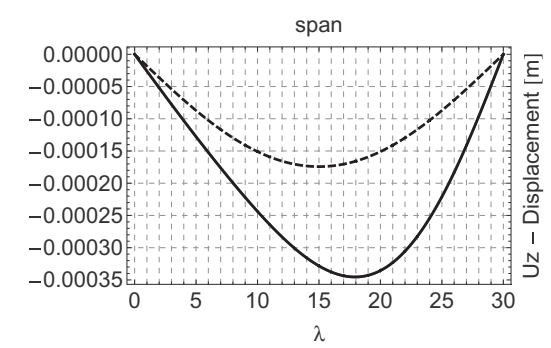

(c)

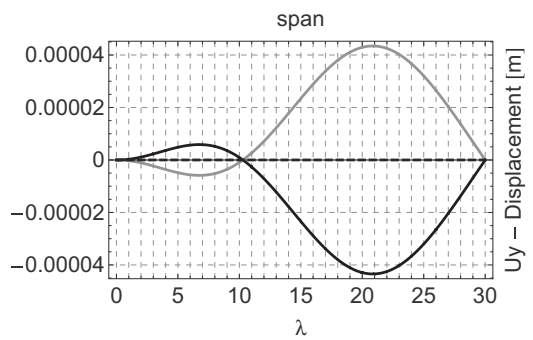

(b)

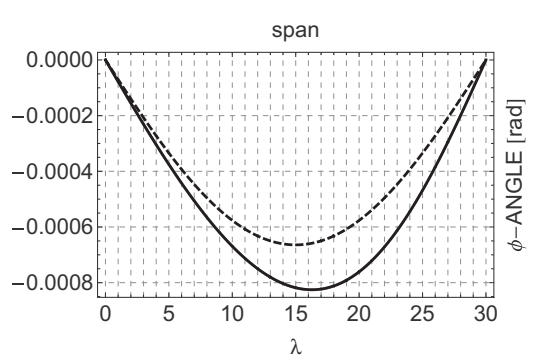

(d)

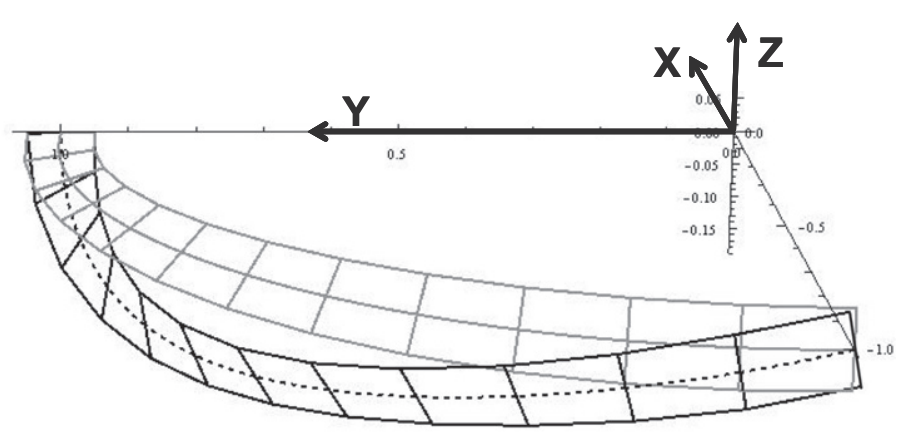

(e)

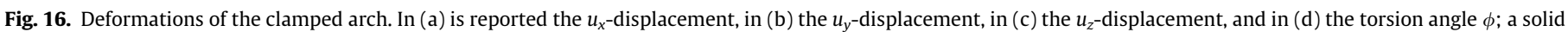

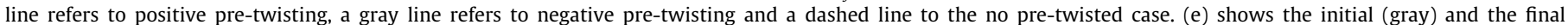
configurations (black) of the pre-twisted rod (in the case of positive twisting) due to a constant vertical distributed load.

$$
\frac{\left\|()_{\text {exac }} \quad()_{\text {num }}\right\|}{\left\|()_{\text {exac }}\right\|} \frac{\sqrt{ } \int_{L_{0}}\left[()_{\text {exac }}()_{\text {num }}\right]^{2} d S}{\sqrt{\int_{L_{0}}()_{\text {exac }}^{2} d S}}
$$

Each curve of the plots is related to a fixed degree of the B spline, and corresponds to an $h$ refinement. In the plots are also shown the lines corresponding to different rates of convergence. It can be ob served that, for the displacements, Fig. 7(a), (b), the asymptotic con vergence rate seems slightly better that the theoretical rate $p+1$ [4]. A transition region appears where the convergence rate is even faster. For comparison, on the same plots has been superposed the error found with Bezier's interpolation, whose convergence is expo nential, since, in this case, increasing the number of control points is equivalent to increase the polynomial degree.

Similar conclusions are drawn from the plots of the rate of con vergence of the error for the stress resultants, compared with the theoretical rate, respectively $p$ for the axial force and the torsional moment and $p \quad 1$ for the bending moments. For this example, also the convergence analysis for the displacement and rotation of the free end, and for the bending and torsional moment at the built in end are presented (Figs. 9(a), (b), 10(a) and (b)). The error for the vertical displacement of the free end is proportional to the er ror in energy. The convergence rate, as expected, is smaller than the one found for the $L^{2}$ norm.

\subsection{D Cantilever arch with a point force}

In this subsection it is considered a flat cantilever arch loaded at the free end by a vertical force. The geometry is the same as in the previous section: the radius of the centroid curve is $R=1[\mathrm{~m}]$ the section is rectangular with $h_{n}=0.1$ and $h_{v}=0.01[\mathrm{~m}]$ respectively, and $\mathrm{E}=1.999 * 10^{8}\left[\mathrm{kN} / \mathrm{m}^{2}\right]$.

The exact solution for the displacements is

$$
\begin{array}{ll}
u_{x}(S) \frac{\operatorname{Sin}[S]^{2}}{2 E \mathcal{I}_{n}}, \\
u_{y}(S) \quad \frac{2 S}{\mathrm{E} \mathcal{A}} \frac{S}{2 \mathrm{E} \mathcal{I}_{n}},
\end{array}
$$

with $S$ the arc length $S \in[0, P i / 2]$, represented in Fig. 11(b) and (c). For $S=P i / 2$ the displacement of the loaded end is

$$
\begin{array}{lrrrr}
u_{x}(\pi / 2) & \frac{1}{2 \mathrm{E} \mathcal{I}_{n}} & 0.30015007503751867 & {[\mathrm{~m}],} \\
& \frac{\pi}{\mathrm{E} \mathcal{A}} & \frac{\pi}{4 \mathrm{E} \mathcal{I}_{n}} & 0.47149035117732546 & {[\mathrm{~m}] .}
\end{array}
$$

In Fig. 12(a) and (b) are presented the $L^{2}$ convergence analy ses of the relative error for the $x$ and $y$ components of the displace ment. The meaning of the plots is the same as in the previous 


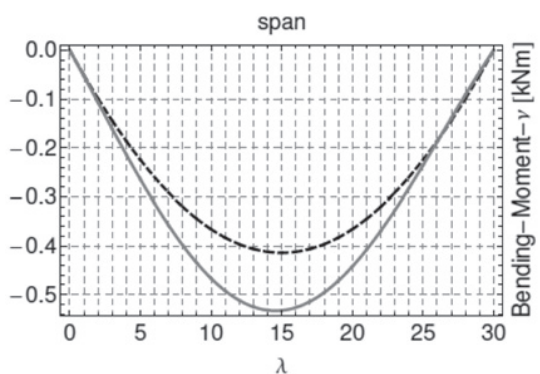

(a)

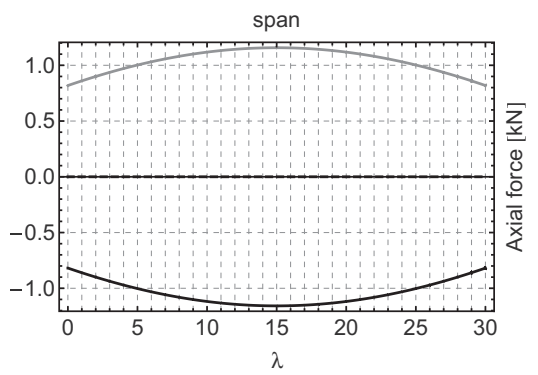

(c)

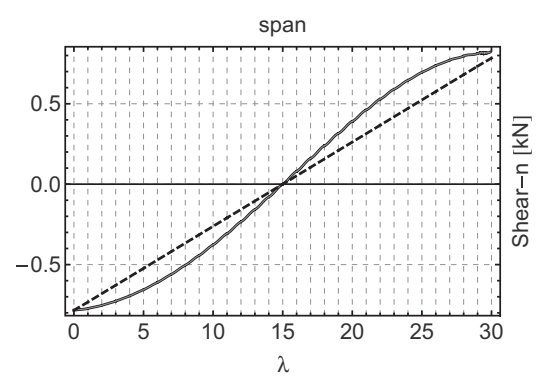

(e)

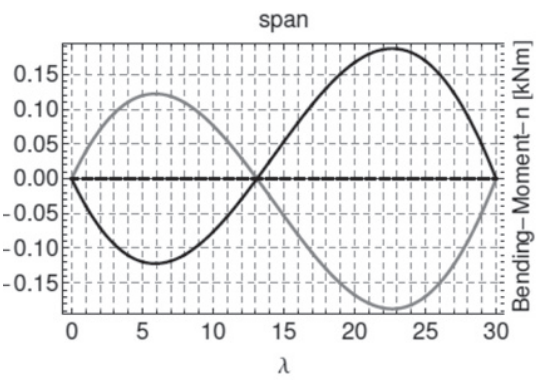

(b)

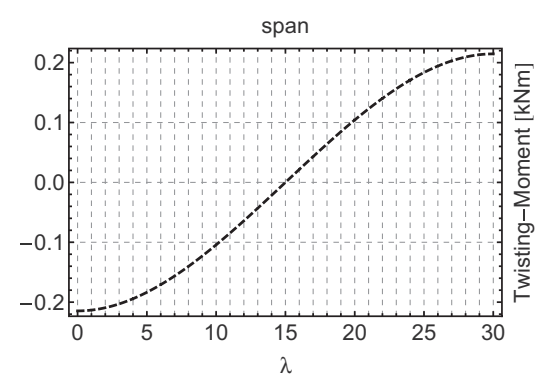

(d)

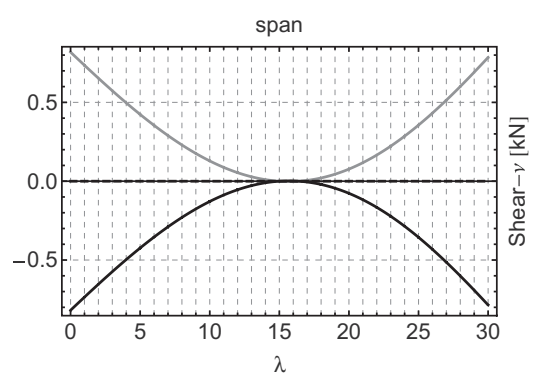

(f)

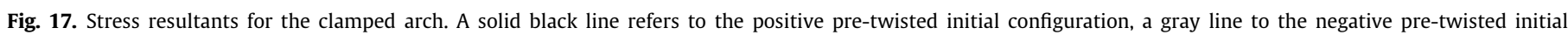
configuration and a dashed line to the torsion free initial configuration.

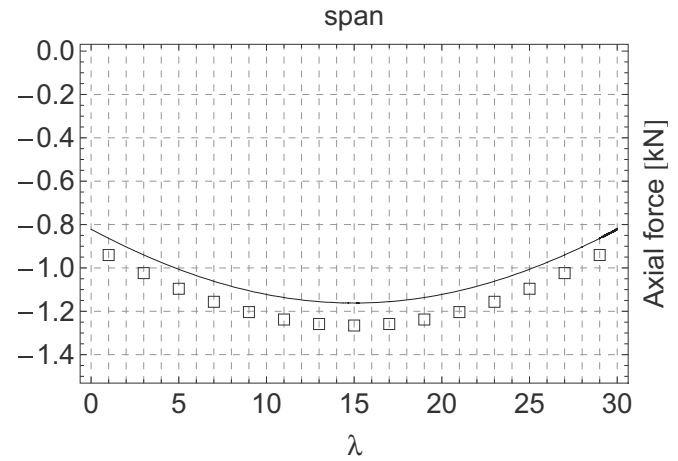

Fig. 18. (FE comparison) Comparison of the axial force obtained with the B-spline interpolation (solid line) and the axial force calculated with 15 Hermitian beam elements (box markers)

example. Also in this example the curves appear to superconverge in the considered range of discretization.

In Fig. 12(a) the dashed curves represents the convergence rate for a $p$ refinement, that is close to the convergence rate of the Be zier's interpolation.

Convergence analysis for the relative error on the bending mo ment, the axial force and the shear are shown in Figs. 13(a), (b) and
Fig. 14(a). An anomalous behaviour is presented by the conver gence of the axial force, that first gets worse then tends to con verge. This behaviour can be explained with the onset of membrane locking. The phenomenon has been already observed Stolarski and Belytschko [25] and will be object of future investigations.

In Fig. 14(b) is presented a comparison between NURBS (isogeo metric) analysis and the present B Spline analysis. In this case the NURBS approximation has the advantage of exactly describing the reference geometry. Furthermore the approximating functions are richer than B splines (as a matter of fact they reduce to B splines when the weights are all unitary). The error on the tip vertical dis placement $u_{y}(\pi / 2)$ under $h$ refinement for two polynomial degrees is presented. As can be observed, increasing the number of ele ments the differences between the two strategies are immaterial.

\subsection{Pre twisted arch}

In this subsection we consider a thin pre twisted arch clamped at both ends, i.e. $\boldsymbol{u}(0)=\boldsymbol{u}(L)=0$ and $\phi(0)=\phi(L)=0$. Three cases are compared, considering positive, null and negative pre twisting. This is a simple case of a non geodetic manifold, because the unit triad if affected by torsion. The centroid curve is given by Eq. (95) with radius $R=1[\mathrm{~m}]$, the section is rectangular with $h_{n}=0.1$ 


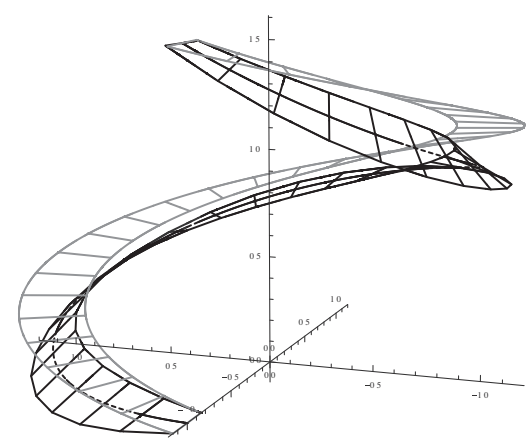

(a)

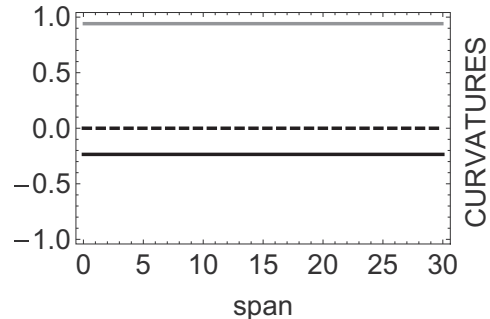

(b)

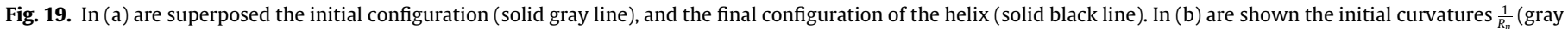
line), $\frac{1}{R_{v}}$ (dashed line) and the initial torsion $\frac{1}{\tau}$ (thick black line).

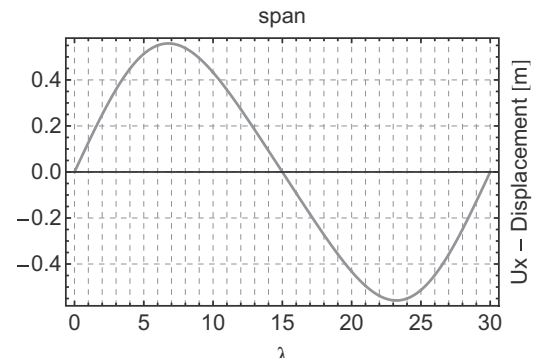

(a)

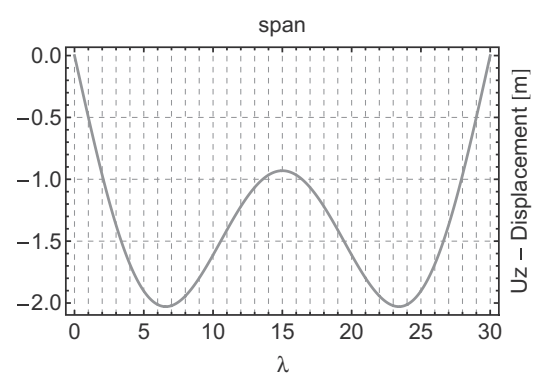

(c)

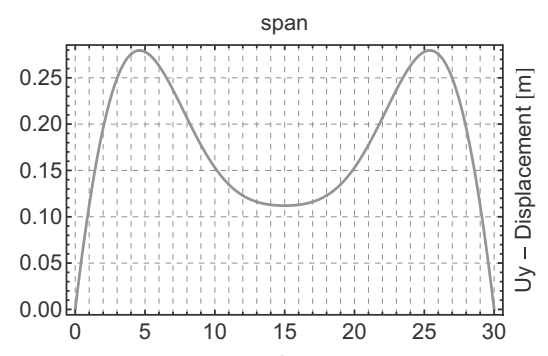

(b)

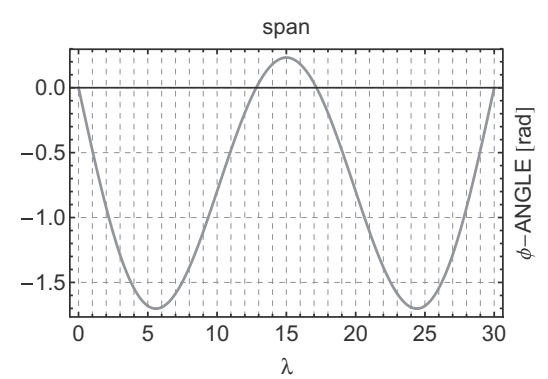

(d)

Fig. 20. Twisted helix. Results for the displacements $u_{x}, u_{y}, u u_{z}$ and for the torsion angle $\phi$.

and $h_{v}=0.01[\mathrm{~m}]$, Young modulus is $\mathrm{E}=1.999 * 10^{8}\left[\mathrm{kN} / \mathrm{m}^{2}\right], v=0.25$ and $G=\mathrm{E} /(2+2 v)$. The initial unit tangent at the origin is $\hat{\boldsymbol{t}}_{0}(0) \quad\{0,1,0\}$ and the initial unit normal vector at the origin is $\hat{\boldsymbol{n}}_{0}(0) \quad\{0,0,1\}$, so that $\hat{\boldsymbol{v}}_{0}(0) \quad\{1,0,0\}$. The initial normal vector is obtained using the rotation operator $\Lambda\left(\hat{\boldsymbol{t}}_{0}(0), \hat{\boldsymbol{t}}_{0}(S)\right)$, that yields

$\hat{\boldsymbol{n}}_{\mathbf{0}}^{\mathrm{b}}(S) \quad \boldsymbol{\Lambda}(\hat{\boldsymbol{t}}(0), \hat{\boldsymbol{t}}(S)) \hat{\boldsymbol{n}}_{\mathbf{0}}(0) \quad \hat{\boldsymbol{n}}_{\mathbf{0}}(0)$

The rotation around $\hat{\boldsymbol{t}}(S)$, is given by the twist angle $\phi$

$\hat{\boldsymbol{n}}_{\mathbf{0}}(S) \quad \boldsymbol{R}\left(\phi_{0}(S), \hat{\boldsymbol{t}}_{\mathbf{0}}(S)\right) \hat{\boldsymbol{n}}_{\mathbf{0}}(0), \quad \hat{\boldsymbol{v}}_{\mathbf{0}}(S) \quad \hat{\boldsymbol{t}}_{\mathbf{0}}(S) \times \hat{\boldsymbol{n}}_{\mathbf{0}}(S)$.

Three cases are considered for the correction angle $\phi_{0}(S)$, as shown in Fig. 15(a), (c) and (e), that is $\phi_{0}(S) \quad \frac{\pi}{2} \frac{S}{L}$ corresponding to an initial configuration with positive constant torsion $\frac{1}{\tau_{0}} 1, \phi_{0}(S) \quad 0$ corresponding to an initial configuration not pre twisted with zero constant torsion $\frac{1}{\tau_{0}} \quad 0$ and $\phi_{0}(S) \quad \frac{\pi}{2} \frac{S}{L}$ cor responding to a negative pre twisted initial configuration with negative constant torsion $\frac{1}{\tau_{0}} \quad 1$.

In Fig. 15(b), (d) and (f), respectively, are plotted the initial con stant torsion and the initial curvatures that are not constant, while
Fig. 15(a), (c), (e) show a sketch of the initial geometries of the arch.

The rod is subjected to a constant distributed vertical force $q_{z}=1[\mathrm{kN} / \mathrm{m}]$. A quintic degree B spline interpolation is consid ered for the initial and deformed configurations.

In Fig. 16(a) (d) are reported the displacements and the torsion angle $\phi$ for the three cases considered (a solid black line relates to the arch with positive twisting, a dashed line to the arch without twisting, and a gray line to the arch with negative twisting).

In Fig. 17(a) (e) and (f) the bending moments, the axial force, the torsional moment and shear forces are shown. It can be noticed that in the case of pre twisted arches because of the coupling be tween flexure and torsion, also a non zero axial force arises, as well as a bending moment around the $\hat{\boldsymbol{n}}$ axis. The results for the axial force are compared in Fig. 18 with those obtained using a Finite Element code, discretizing the arch with 15 straight Hermitian ele ments, model that requires a great amount of modeling effort. The FE results are less accurate due to the geometrical errors in inter polating the tangent to the rod. 


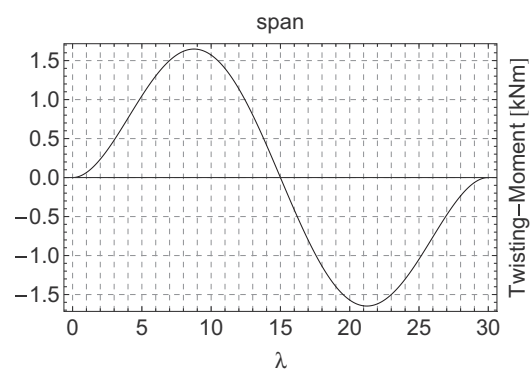

(a)

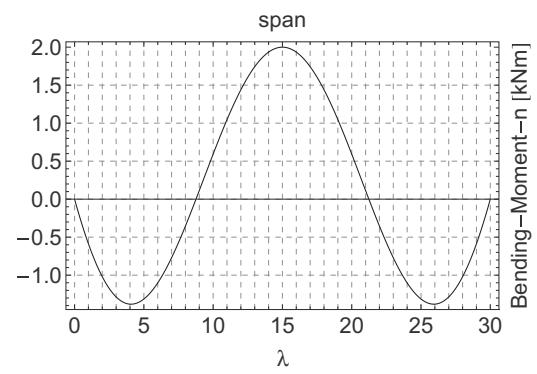

(c)

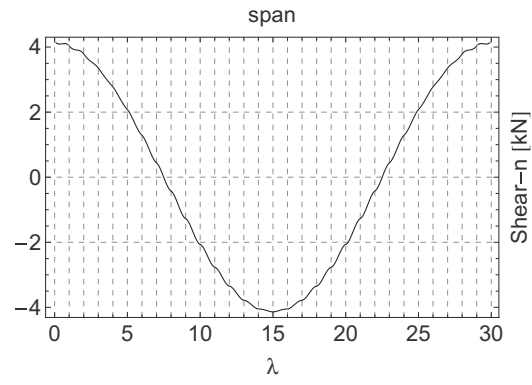

(e)

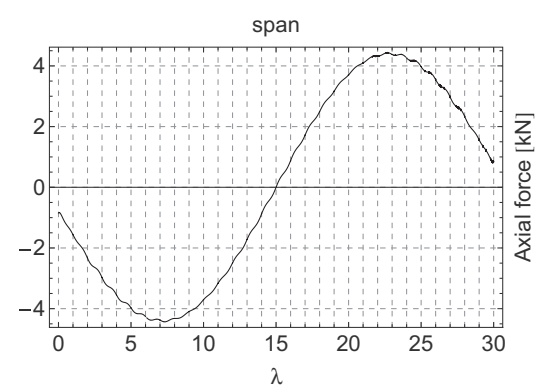

(b)

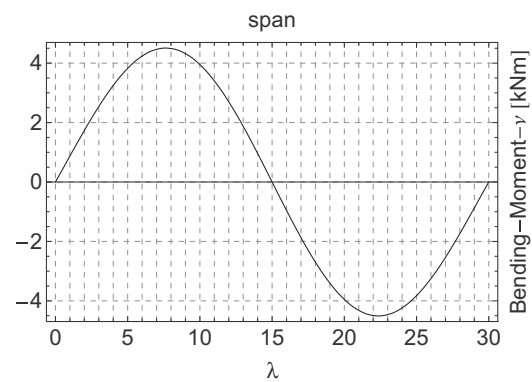

(d)

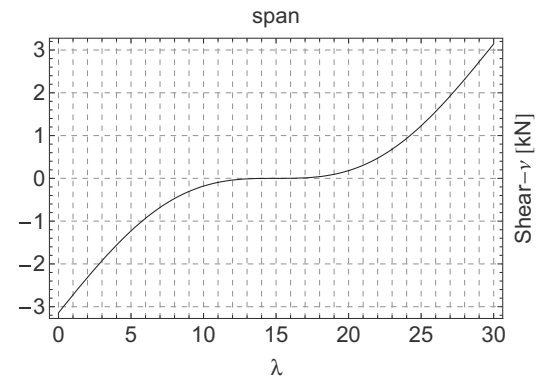

(f)

Fig. 21. Twisted helix. Results for the stress resultants.

\subsection{General case: pre twisted cylindrical helix}

In this sub section we consider a rod with a centroid curve de fined by an helix, mapped by the expressions

$P_{x}(\alpha) \quad R \operatorname{Cos}\left[\begin{array}{ll}\pi & \alpha\end{array}\right], \quad P_{y}(\alpha) \quad R \operatorname{Sin}\left[\begin{array}{ll}\pi & \alpha\end{array}\right]$,

$P_{z}(\alpha) \quad p \alpha, \quad \alpha \in[0,2 \pi]$,

with $R=1[\mathrm{~m}]$ and $p=0.25[\mathrm{~m}]$. The unit normal, $\hat{\boldsymbol{n}}$, is mapped on the cartesian bases, $\boldsymbol{E}_{i}$, as

$$
\begin{aligned}
& \hat{\boldsymbol{n}}_{\mathbf{0}} \cdot \boldsymbol{E}_{x}(\alpha) \quad \operatorname{Cos}\left[\begin{array}{ll}
\pi & \alpha
\end{array}\right], \quad \hat{\boldsymbol{n}}_{\mathbf{0}} \cdot \boldsymbol{E}_{y}(\alpha) \\
& \operatorname{Sin}\left[\begin{array}{ll}
\pi & \alpha
\end{array}\right], \quad \hat{\boldsymbol{n}}_{\mathbf{0}} \cdot \boldsymbol{E}_{z}(\alpha) \quad 0, \quad \alpha \in[0,2 \pi],
\end{aligned}
$$

and coincides with the unit normal to the cylinder in the points on which the helix lies. The third unit vector $\hat{v}$ is mapped by $\hat{\boldsymbol{v}}_{\mathbf{0}} \quad \hat{\boldsymbol{t}}_{\mathbf{0}} \times \hat{\boldsymbol{n}}_{\mathbf{0}}$.

The thickness of the rectangular section are $h_{n}=0.1[\mathrm{~m}]$ and $h_{v}=0.01[\mathrm{~m}]$ the Young's modulus is $E=1.999 \times 10^{8}\left[\mathrm{kN} / \mathrm{m}^{2}\right]$, $v=.25$ and $G=\mathrm{E} /(2+2 v)$. The initial configuration, and the initial curvatures and twist are shown in Fig. 19(a).

The boundary conditions are $u_{x}(0)=u_{y}(0)=u_{z}(0)=\phi(0)=0$ and $u_{x}(L)=u_{y}(L)=u_{z}(L)=\phi(L)=0$. The external force is $q=\{0,0,1\}$ $[\mathrm{kN} / \mathrm{m}]$, proportional to the self weight. The rod is modeled by a quintic B spline with 35 internal points. The displacement $u_{x}, u_{y}, u_{z}$ and the rotation $\phi$ are shown, respectively, in Fig. 20(a) (d). The stress resultants are reported in Fig. 21(a) (f).

The plots of the stress resultants are quite smooth, as was ex pected, particularly for the shears, that are the most sensitive to er rors and oscillations.

\section{Conclusions}

In the paper has been investigated a numerical method for the analysis of curved and twisted elastic space rods, subjected to gen eral loading conditions. The numerical treatment of this kind of structures in the context of standard finite element approxima tions requires a great modelling effort, and the results suffer of sig nificant inaccuracy due to the geometrical discontinuities brought about by the FE discretization. Therefore it has been proposed an approach based on B spline approximation, that allows to describe with high continuity and with asymptotic precision the initial geometry.

Kirchhoff Love rods have been analyzed, since in the case of thin rods (the ones examined in the article) shear locking is avoided (but not, in general, membrane locking, as one of the example presented suggests). First it has been developed a 3D model for the deformation of the rod, defined as a ribbon extruded over a base line. The evolution of the section of the rod along the 
axis has been defined by means of a rotation operator that does not use Euler angles, and that has proved to be quite convenient. The tangent kinematic operator has been derived in a Lagrangian description. An updated Lagrangian form of the equilibrium equa tions has been obtained, from which the definitions of the reduced axial and shear forces often used in the literature naturally stem out. In the hypothesis of a linear elastic behaviour we have derived the constitutive equations of the rod in terms of the effective stress resultants.

Next the linearized equilibrium equations have been stated, in the hypothesis of infinitesimal deformations. The applications pre sented are limited to this case.

An interpolation based on B spline bases has been considered. The material stiffness matrix has been explicitly derived using a single patch for the rod. The stiffness matrix is highly coupled, and the axial, bending and torsional contributions have been pre sented separately. The geometric and the load stiffness matrices will be presented in a future work. An isoparametric approach has been used, employing the same B splines for the reference geometry and for the configuration variables. By means of an aca demic example, it has been shown that, for the case of spatial rods, the approach based on NURBS has no significant improvement on the proposed approach based on B splines.

Convergence analyses on simple examples yield convergence rate close to the theoretical ones, and even better in a transition re gion. Also the convergence rate for $k$ refinement has been tested.

The examples presented have proved the efficiency of the meth od proposed. At least in one of the examples presented locking phenomena have been observed. Indeed locking may be present for very thin sections. However this important problem has not been addressed explicitly in the present work and will be object of a forthcoming paper.

\section{References}

[1] S.S. Antman, Nonlinear Problem of Elasticity, Springer-Verlag, New York, 1995

[2] F. Armero, J. Valverde, Invariant hermitian finite elements for thin kirchhof rods. ii: The linear three-dimensional case, Comput. Methods Appl. Mech. Eng. (2012) 458-485.

[3] F. Auricchio, F. Calabró, T.J.R. Hughes, A. Reali, G. Sangalli, A simple algorithm for obtainig nearly optimal quadrature rule for nurbs-based isogeometric analysis, Comput. Methods Appl. Mech. Eng. (2012). <http://dx.doi.org/1016/ j.cma.2012.04.014>.
[4] Y. Bazilevs, L.B. ao Da Veiga, J.A. Cottrell, T.J.R. Hughes, G. Sangalli, Iogeometric analysis: approximation, stability and error estimates for h-refined meshes, Math. Models Methods Appl. Sci. 16 (2006) 1031-1090.

[5] D. Benson, Y. Bazilevs, M. Hsu, T. Hughes, Isogeometric shell analysis: the reissner-mindlin shell, Comput. Methods Appl. Mech. Eng. (2010) 276-289.

[6] D. Benson, Y. Bazilevs, M. Hsu, T. Hughes, A large deformation, rotation-free, isogeometric shell, Comput. Methods Appl. Mech. Eng. (2011) 1367-1378.

[7] M. Bergou, M. Wardetzky, S. Robinson, B. Audoly, E. Grinspun, Discrete elastic rods, ACM Trans. Graphics 27 (2008) 63:01-63:12.

[8] K.-U. Bletzinger, M. Bischoff, E. Ramm, A unified approach for shear-locking free triangular and rectangular shell finite elements, Comput. Struct. 75 (2000) 321-334.

[9] J. Cottrell, T. Hughes, A. Reali, Studies of refinement and continuity in isogeometric structural analysis, Comput. Methods Appl. Mech. Eng. 196 (2007) 4160-4183.

[10] M.A. Crisfield, A consistent co-rotational formulation for non-linear, threedimensional, beam elements, Comput. Methods Appl. Mech. Eng. 81 (1990) 131-150.

[11] R. Echter, M. Bischoff, Numerical efficiency, locking and unlocking of nurbs finite elements, Comput. Methods Appl. Mech. Eng. 199 (2010) 374-382.

[12] C. Gontier, C. Vollmer, A large displacement analysis of beam using a cad geometric definition, Comput. Struct. 57 (1995) 981-989.

[13] A. Goriely, M. Nizette, M. Tabor, On the dynamic of elastic strips, J. Nonlinear Sci. 11 (2001) 3-45.

[14] T. Hughes, J. Cottrell, Y. Bazilevs, Isogeometric analysis: cad, finite elements, nurbs, exact geometry, and mesh refinement, Comput. Methods Appl. Mech. Eng. 194 (2005) 4135-4195.

[15] T. Hughes, A. Reali, G. Sangalli, Efficient quadrature for nurbs-based isogeometric analysis, Comput. Methods Appl. Mech. Eng. 199 (2010) 301313.

[16] M. Ishaquddin, P. Raveendranath, J. Reddy, Flexure and torsion locking phenomena in out-of-plane deformation of timoshenko curved beam element, Finite Elem. Anal. Des. 51 (2012) 22-30.

[17] J.A. Cottrell, Y. Bazilevs, T.J. Hughes, Isogeometric Analysis: Toward Integration of CAD and FEA, Wiley, 2009.

[18] J. Kiendl, K.-U. Bletzinger, J. Linhard, R. Wuchner, Isogeometric shell analysis with Kirchoff-Love elements, Comput. Methods Appl. Mech. Eng. 189 (2009) 3902-3914.

[19] J. Kiendl, Y. Bazilevs, M.-C. Hsu, R. Wuchner, K.-U. Bletzinger, The bending strip method for isogeometric analysis of Kirchhoff-Love shell structures comprised of multiple patches, Comput. Methods Appl. Mech. Eng. 199 (2010) 24032416.

[20] J. Langer, D. Singer, Lagrangian aspects of the Kirchhoff elastic rod, SIAM Rev 38 (1995) 605-618.

[21] L. Piegl, W. Tiller, The NURBS Book, 2nd ed., Springer-Verlag, New York, 1995.

[22] J. Simo, A finite strain beam formulation. The three dimensional dynamic problem. Part i, Compt. Methods Appl. Mech. Eng. 42 (1986) 55-70.

[23] J. Simo, D. Fox, On a stress resultant geometrically exact shell model. Part i: Formulation and optimal parametrization, Comput. Methods. Appl. Mech. Eng. 72 (1989) 267-304.

[24] J.C. Simo, L. Vu-Quoc, A three dimensional finite strain rod model. Part ii: Computational aspect, Comput. Methods. Appl. Mech. Eng. 58 (1986) 79-116.

[25] H. Stolarski, T. Belytschko, Shear and membrane locking in curved c0 elements, Comput. Methods Appl. Mech. Eng. 41 (1983) 279-296. 